\title{
Der Wille und das räumliche Moment in Wahrnehmung und Vorstellung.
}

Von

Dr. E. Storch,

Privatdocent an der Universität Breslau.

(Mit 12 Textfiguren.)

In meinem Aufsatze „Psychologie und Medicin ${ }^{1}$ ) habe ich die Anschauung bekämpft, dass die Wahrnehmung eines Gegenstandes darin bestehe, dass der sinnliche Reiz, welcher die Wahrnehmung auslöst, eine grössere Anzahl von Theilvorstellungen, optischen, tactilen, geustischen, akustischen Erinnerungsbildern des Objectes wachruft, deren Summe uns eben als Object im Raume bewusst ist.

Auf Grund der Thatsache; dass in allen sinnlichen Wahrnehmungen ein und dieselbe Bewusstseinserscheinung, die Vorstellung von dem Ort des Objectes, wiederkehrt, kamen wir zu dem Schluss, dass das räumliche Moment aller unserer Wahrnehmungen auf dem gleichen materiellen Process in der Grosshirnrinde beruhen müsse, dass wir ein eigenes nervöses Organ besitzen, dessen Erregung uns als Raumvorstellung zum Bewusstsein kommt.

Dieses nervöse, der Raumvorstellung dienende Organ nannte ich stereopsychisches Feld, seine anatomischen Elemente Stereone. Da die Erregungen aller Sinnescentren adäquate Reize des stereopsychischen Feldes darstellen, und da andererseits jede Raumvorstellung, wenn sie als räumliches Moment des Bewegungswillens erscheint, auch das motorische Neuronsystem der Grosshirnrinde zu innerviren vermag, so wurzeln die Stereone mit ihren Dendriten in den verschiedenen Sinnescentren und reichen mit ihren Neuriten in die motorische Zone hinein.

Das stereopsychische Feld erstreckt sich demnach über den grössten Theil der gesammten Hirnrinde. An verschiedenen, mehr

1) Arch. f. d. ges. Physiol. Bd. 93 S. 412.

E. Pflüg er, Archiv für Physiologie. Bd. 95. 
weniger abgegrenzten Stellen liegen darin eingesprengt die verschiedenen pathopsychischen und motorischen Neuronsysteme. Solange der Stoffwechsel in allen Theilen des stereopsychischen Feldes unverändert bleibt, d. h. solange in jedem Stereon die den Gesammtstoffwechsel ausmachenden Theilchemismen mit unveränderter Geschwindigkeit ablaufen, solange findet keinerlei Aenderung unserer räumlichen Vorstellungen statt. Sobald aber aus irgend welchen Ursachen, sei es in einem Theile der Stereone, sei es in ihrer Gesammtheit, eine der möglichen Veränderungen stattfindet, tritt auch irgend eine Veränderung unserer Raumvorstellungen ein.

Ein feineres Reagens für die Veränderungen des stereopsychischen Chemismus als unser Bewusstsein gibt es nicht. Die Veränderungen unserer Raumvorstellungen sind das Erleben der chemischen Veränderungen im stereopsychischen Felde. Wenn wir demnach unsere Raumvorstellungen analysiren, d. h. wenn wir untersuchen, was ihnen allen gemeinsam ist, und wodurch sie sich unterscheiden, so untersuchen wir zu gleicher Zeit auch die Gemeinsamkeiten und Verschiedenheiten der ihnen zu Grunde liegenden stereopsychischen Chemismen.

Diese Analyse führte uns zu der Ueberzeugung, dass alle unsere Raumvorstellungen aus der Verbindung dreier psychischer Elemente hervorgehen, deren jedes seiner Intensität nach unabhängig von den beiden anderen veränderlich ist.

Wie gesagt, irgendwie vorstellbar ist ein solches Element nicht, weil wir den ihm zu Grunde liegenden stereopsychischen Theilchemismus niemals für sich erleben. Aber wir haben bei der Vergleichung des einund zweiäugigen Sehactes gesehen, wie ein solches Flement zu den schon vorhandenen zwiefach unendlichen Beziehungen eine dritte unendliche Mannigfaltigkeit von Bestimmungen hinzufügt. Während beim einäugigen Sehact in völlig unbekannter Umgebung nur eine Anordnung der Lichtpunkte in Bezug auf Oben, Unten, Rechts und Links erfolgt, ordnen wir beim zweiäugigen Sehen die Sehdinge auch der Tiefe nach an.

Es ist meiner Ueberzeugung nach diese längst bekannte Thatsache selbst in Kreisen der Psychologen nicht hinreichend gewürdigt worden. Man muss sich in der That den Unterschied zwischen der sinnlichen Tiefenwharnehmung beim zweiäugigen Sehen, und der Raumwahrnehmung beim einäugigen Sehen, der jede Anordnung in der Tiefe fehlt, durch eigene Anschauung klarmachen, 
Der Wille u. das räumliche Moment in Wahrnehmung u. Vorstellung. 307

um die Bedeutung dieser Erscheinung für die Theorie der Sinneswahrnehmungen zu erfassen.

Am Besten geschieht das, wenn man durch ein Loch an der Thüre eines Dunkelzimmers eine Anzahl mattleuchtender Scheiben, die in verschiedenen Entfernungen vom Beschauer angebracht sind, zunächst einäugig und dann zweiäugig betrachtet. Man bildet sich dann zwar zuerst ein, Unterschiede in der Entftrnung wahrzunehmen, erkennt aber, sobald man mit zwei Augen beobachtet, dass man von der wirklichen Tiefenanordnung keine Ahnung hatte.

Nun belehrt uns aber eine einfache Umfrage bei unbefangenen Leuten, wie kaum Einer von selbst bemerkt, dass sich bei Schluss eines Auges das Aussehen seiner Umgebung ändert. Jeder glaubt mit Sicherheit auch die Tiefenerstreckung der gesehenen Dinge genau so aufzufassen wie beim Sehen mit zwei Augen. Wenn nun der einäugige Sehact an sich, wie feststeht, diese Kenntniss nicht vermitteln kann, so müssen sich bei einäugiger Betrachtung bekannter Dinge Bewusstseinsvorgänge abspielen, die mit dem Wahrnehmungsprocess nur in einem mittelbaren Zusammenhang stehen, Bewusstseinsvorgänge, die erst auftreten können, wenn ich die wirkliche Gestalt der Sehdinge, ihre Tiefenerstreckung kenne. Diese Kenntniss kann mir aber, wie gezeigt, nicht aus den einäugigen Seherfahrungen gekommen sein, sondern aus irgend welchen anderen Sinneswahrnehmungen, z. Th. jedenfalls aus dem zweiäugigen Sehen.

Sehe ich mit einem Auge einen Würfel, so ist das räumliche Moment, das der optische Reiz vor aller Erfahrung in mein Bewusstsein hebt, sicher nicht die Vorstellung des regelmässigen Sechsflächners, sondern es dürfte - freilich ist dieser Vergleich nur eine Annäherung - etwa das nämliche sein, das der Biinde bei Betasten einer erhabenen perspectivischen Würfelzeichnung erhält.

Aus der Erfahrung aber weiss ich, dass dieses räumliche Moment der einäugigen Sehwahrnehmung, die Sehform des Würfels, von einem wirklichen Würfel stammt. Fasse ich nach dem Orte, wo sich der Würfel befindet, und betaste ihn, so ist das räumliche Moment dieser Tastwahrnehmung die wirkliche Würfelform, und die Vorstellung dieser Form ist es, welche jedes Mal, wenn die einäugige Sehform des Würfels dem Erfahrungslosen bewusst werden würde, in das Bewusstsein des Erwachsenen tritt.

Auf diese erst durch die Erfahrung auf anderweitigen Sinnesgebieten mögliche Verdrängung der einäugigen Sehform der Gegen- 
stände durch ihre wirkliche Form möchte ich ganz besonders die Aufmerksamkeit lenken. Zwar ist die Kenntniss der Perspective durchaus nicht jungen Datums, aber trotzdem scheint es mir, als ob diese Kenntniss die ihr in der Psychologie gebührende Würdigung noch lange nicht gefunden hätte.

Ein Blick in die Ecke eines Zimmers, wo die Decke mit zwei Seitenwänden zusammenstösst, belehrt mich, auch bei einäugiger Betrachtung, dass dort drei ebene Flächen unter rechten Winkeln zusammenstossen, und es kommt mir bei unbefangener Betrachtung gar nicht zum Bewusstsein, dass es eigentlich gar nicht drei rechte Winkel sind, die ich streng genommen "sehe", dass ich eigentlich nur auf Grund meines anderweitig gewonnenen Wissens von der wirklichen Form dieser Ecke für drei Winkel, deren Summe vier $\mathrm{R}$ beträgt, die Vorstellung der dreiseitig rechtwinkligen Ecke einsetze.

Die rechteckige Tischplatte, an welcher ich schreibe, erscheint mir auch bei einäugiger Betrachtung ohne Weiteres als Rechteck, und doch ist die Sehform derselben keineswegs ein Rechteck. Was diese Vorstellung der'wirklichen Form an Stelle der Sehform in meinem Bewusstsein auftauchen lässt, ist wiederum nur die Erfahrung, meine anderweitig gewonnene Kenntniss der wirklichen Form.

Es ist nicht ohne Interesse, zu beobachten, wie die Menschen im unbefangenen Zustande, die Völker des früheren Altertums, die Wilden, und unsere Kinder bei ihrem Zeichenversuchen immer wieder beweisen, dass der stereopsychische Process, welcher unmittelbar durch den Sehreiz ausgelöst wird, eigentlich gar nicht zum Bewusstsein kommt, sondern nur als Agent provocateur der wirklichen Formen dient. Eine grosse Zahl kleiner Kinder zeichnet das menschliche Gesicht, das einen beliebten Vorwurf darstellt, so, dass die Nase als Vorsprung an der Seite sichtbar ist, also im Profil; in den Kreis aber, der das Gesicht darstellt, werden neben einander die beiden Augen gesetzt, die der Mensch ja wirklich besitzt, und darunter der Mund als wagerechter Strich, denn als solcher wird er ja wirklich gefühlt.

Nie werden solch kleine Künstler es unterlassen, einem Säugethiere auf der Zeichnung die vier ihm zukommenden Beine angedeihen $\mathrm{zu}$ lassen, und oft zeichnen sie den Leichnam in den verschlossenen Sarg; die Bewohner, Tische, Stühle u. s. w. in ein von Aussen gesehenes Haus.

Und Aehnliches, wie gesagt, findet man bei den alten Culturvölkern, den Aegyptern, Babyloniern, Assyrern. Immer sind diese 
Der Wille u. das räumliche Moment in Wahrnehmung u. Vorstellung. 309

Zeichnungen ein Beweis dafür, dass keineswegs das, was eigentlich „gesehen" wird, sondern die wirkliche Form der Dinge durch den Sehact ins Bewusstsein gehoben wird.

Was ich hier vom einäugigen Sehen gesagt habe, gilt nun auch, wenn schon in etwas abgemilderter Form, vom zweiäugigen Sehen. Durch Untersuchungen L. Heine's ist es festgestellt, dass von zwei Punkten im Raum, $A$ und $B$, bei doppeläugiger Fixation des einen, $A$, der andere, $B$, noch merklich vor oder hinter $A$ wahrgenommen wird, wenn seine beiden Netzhautbilder $\beta_{l}$ und $\beta_{r}$ eine Querdisparation von mindestens fünf Bogensecunden haben. Wird die Querdisparation geringer, so hört jede Tiefenwahrnehmung auf. Auf Grund dieser für die meisten Normalsichtigen zutreffenden Bestimmung lässt sich ausrechnen, welche Tiefenerstreckung $x$ zwischen $B$ und $A$ liegen muss, damit bei einer Entfernung $d$ des dem Beschauer nächsten Punktes $A$ noch eine Tiefenwahrnehmung zu Stande kommt; $x$ ist mit für die Praxis hinreichender Genauigkeit gleich $d^{2}:(2000-d)$ Meter.

Daraus ergibt sich, dass wir die dritte Dimension eines Gegenstandes noch sehen, wenn dieser bei einer Entfernung $d$ eine Tiefenausdehnung von mindestens $x$ Metern hat:

$\begin{array}{ll}d=0,4 \mathrm{~m} & x=0,00008 \mathrm{~m} \\ d=1 & x=0,0005 \quad " \\ d=2 & x=0,002 \quad " \\ d=3 & x=0,0045 \quad " \\ d=10, & x=0,05 \quad " \\ d=100 " & x=5,0 \quad \\ d=1000 " & x=1000 \quad \\ d=2000, & x=\infty\end{array}$

D. h.: Schon in einer Entfernung von $10 \mathrm{~m}$ muss ein Object, um körperlich gesehen zu werden, mindestens $5 \mathrm{~cm}$ Tiefenausdehnung haben, bei $100 \mathrm{~m}$ Entfernung aber schon $5 \mathrm{~m}$, und bei $2 \mathrm{~km} \mathrm{En}$ fernung hört alles körperliche Sehen auf. Die wirklich "gesehene" Tiefenerstreckung $x$ eines Objectes nimmt also sehr rasch mit seiner Entfernung ab (bis.zu $100 \mathrm{~m}$ etwa im umgekehrten Quadrat der Entfernung), jedenfalls viel rascher als die Grösse des Netzhautbildes einer in der Bildebene gelegenen Linie.

Ich habe gefunden, dass man etwa in einer Entfernung zwischen 30 und $50 \mathrm{~cm}$ Tiefenerstreckungen und Abstände in der Bildebene für gleich gross schätzt, wenn sie wirklich gleich gross sind. Jedenfalls muss es theoretisch eine bestimmte Entfernung geben, in welcher 
ein Würfel in seiner wirklichen Form gesehen wird. Nehmen wir also an, ein Würfel von der Seitenlänge $a=1$ sei in dieser Entfernung, also $40 \mathrm{~cm}$ von der Nasenwurzel des Beschauers so aufgestellt, dass eine seiner Fläehen in die Bildebene fällt; dann sehe ich, den Würfel als durchsichtig angenommen, die hintere Fläche in der Entfernung $a$ von der vorderen.

In der Entfernung von $1 \mathrm{~m}$ hat das Netzhantbild der Kanten der vorderen Fläche sich auf 0,4 des ursprünglichen Betrages verringert, dagegen hat die Querdisparation der Netzhautbilder eines hinteren Eckpunktes nur noch $0,4^{2}$ ihres ursprünglichen Werthes. Setze ich die gesehene Seitenlänge des vorderen Quadrates nunmehr wieder $\mathbf{1}=a$, so ist die wirklich gesehene Entfernung der vorderen von der hinteren Würfelfäche nur noch 0,4 . Ich sehe den Würfel also nicht mehr in seiner wirklichen Form, sondern zusammengedrückt, als Relief.

Bei $2 \mathrm{~m}$ wäre, die wirklich gesehene Seitenlänge des vorderen Quadrates immer gleich 1 gesetzt, die gesehene Tiefenerstreckung nur noch 0,2 , bei $3 \mathrm{~m}$ nur noch 0,13 und bei $10 \mathrm{~m}$ nur 0,04 .

Man sieht hieraus, wie schnell die Dinge mit zunehmender Entfernung für den Sehact ihre Tiefenausdehnung verlieren. Schon in einer Entfernung von $10 \mathrm{~m}$ müssten sie als Basreliefs von $1 / 25$ ihrer wirklichen Tiefe wahrgenommen werden, wenn eben nicht in unserem Bewusstsein die Sebform von der wirklichen Form verdrängt würde.

Also weder beim einäugigen noch beim zweigäugigen Sehen ist die räumliche Anordnung und Form der Dinge, die wir wirklich wahrnehmen, lediglich die subjective Seite des durch den Sebreiz erzeugten stereopsychischen Processes. Ganz ohne unseren Willen tritt an Stelle der Sehform die wirkliche Form der Objecte.

Nur durch eine mühsame und langdauernde Uebung gelingt es uns diesen von aller Erfahrung unabhängigen stereopsychischen Process, wenigstens in der Form, wie er beim einäugigen Sehen zu Stande kommt, in allen Einzelheiten klar in's Bewusstsein zu heben. Beim perspectivischen Zeichnen lernen wir die Begrenzungslinien der Gegenstände in die Bildebene zu projiciren, und erst dadurch wird uns klar, was wir eigentlich "sehen" und wie viel wir aus dem Schatze unserer Erfahrungen zu dem Gesehenen hinzuthun.

Das räumliche Moment der Sehwahrnehmung muss daher in zwei Componenten zerlegt werden. Die eine, unabhängig von aller Erfahrung, die ich als Sehform bezeichnete, entsteht allein 
Der Wille u. das räumliche Moment in Wahrnehmụng u. Vorstellung. 311

durch den Sehreiz, indem dieser lediglich unserer Organisation gemäss zunächst im optischen Lichtfelde der Hinterbauptsrinde und sodann im stereopsychischen Felde eine in allen Einzelheiten gesetzmässig bestimmte Erregung erzeugt, die uns als gewisser Richtungscomplex zwei- oder drei-dimensionaler Natur bewusst wird. Diesen Theil des räumlichen Momentes beim Sehact bezeichne ich als die sinnlich gestützte Raumcomponente oder auch als das unmittelbare räumliche Moment der Sehwahrnehmung.

Dieser sinnlich gestützte Antheil wird nun beim Sehen, wie ich ausgeführt habe, fast völlig unterdrückt durch einen anderen Richtungscomplex, die wirkliche Form der Dinge. Diese letztere im Bewusstsein jedes Erwachsenen stets vorwiegende Raumvorstellung bezeichne ich als mittelbares räumliches Moment der Sehwahrnehmung oder auch als associative Componente.

Es ist klar, dass wir, ausschliesslich auf unsere Sehwahrnehmungen angewiesen, niemals zu der Vorstellung der wirklichen Objectform gelangen könnten. Ein Würfel erscheint, je nach den unendlich vielen Standpunkten, von dem aus wir ihn sehen können, in unendlich vielen verschiedenen Sehformen. Nichts würde uns veranlassen, eine dieser Formen vor der anderen zu bevorzugen, alle wären völlig gleichberechtigt, und zum Begriffe des Objectes würde uns keineswegs die Unveränderlichkeit der Form gehören. Wir würden bei einem Trinkglase von gewöhnlicher cylindrischer Form annehmen, dass es, wenn bewegt, seine Form verändert, dass es vom Rechteck zum Kreis alle möglichen Umwandlungen erfahren kann.

Diese. Annahme wird thatsächlich durch die Erfahrung gestützt. Ich hatte Gelegenheit, in der kgl. Universitätsaugenklinik des Herrn Geh. Uhth off zu Breslau zwei Fälle von frühzeitig eingetretener Blindheit $\mathrm{zu}$ beobachten, die nach langen Jahren durch glückliche operative Eingriffe wiedèr sehend wurden ${ }^{1}$ ).

Besonders der eine dieser Fälle ist bemerkenswerth, weil dle Intelligenz des Patienten eine genauere Untersuchung gestattete. Dieser Kranke war 24 Jahre blind gewesen und hatte fast jede Erinnerung an optisch gewonnene Raumvorstellungen verloren. Er vermochte nicht ein Eichenblatt zu beschreiben, gab die Grösse eines Sperlings gleich der einer Gans an u. s. w. Gleich nach der ersten Abnahme

1) Klin. Monatsbl. f. Augenheilkunde, 40. Jahrg. S. 97. F. Seydel, Ein Beitrag zum Wiedersehenlernen Blindgeborener. 
des Verbandes vermochte er ein Strabometer vom Anblick kenntlich nachzuzeichnen. Er erbielt also durch den Sehact unzweifelhaft Raumvorstellungen.

Besonders bemerkenswerth aber waren seine Aeusserungen über die Wahrnehmung ihm vorgezeigter Gegenstände. Er erkannte die meisten nicht. Eine Visitenkarte erklärte er als Viereck; wurde sie um die Achse gedreht, so meinte er, sie würde schmäler, wurde sie von ihm entfernt, sie würde kleiner. Wir, die gelernt haben, für die Sehform die wirkliche Form zu setzen, nehmen in solchen Fällen nicht Form-, sondern Stellungsveränderungen wahr.

Der Kranke verhielt sich also genau so, wie wir es theoretisch erwarten müssen in Fällen, wo die associative Raumcomponente beim Sehen fehlt. Ganz gleichen Erscheinungen begegnen wir in manchen Fällen von Seelenblindheit, und theoretisch muss diese ja immer eintreten, sobald die Sehform nicht durch die wirkliche Form verdrängt wird. Ich verweise in dieser Beziehung auf die Lissauersche Beobachtung (Arch. f. Psych. Bd. 21 S. 222), ferner auf eine Arbeit Fritz Hartmann's (Wien. klin. Wochenschr. XV Nr. 21 S. 547). Besonders in letzterer hat die verständnissvolle Untersuchung überzeugend nachgewiesen, dass dem seelenblinden Kranken häufig nur die Sehform zum Bewusstsein kommt, und dass seine Unfähigkeit, Objecte optisch zu erkennen, ganz vorwiegend auf dem Fehlen der associativen Raumcomponente beruht. Ich erwähne einige besonders charakteristische Beispiele: „Ein Trinkglas (mit kreisförmigem Querschnitt), mit dem Boden gegen den Beschauer gekehrt, wird vom Patienten als Glas bezeichnet, ohne dass er anzugeben vermöchte, welcher Art das Glas ist, zu welchem Zwecke es dient; er kann nur sagen, dass es rund ist (Erkennung der Begrenzungsform).

„Dasselbe Glas wird nun dem Patienten aufrecht vorgehalten. Patient äusserte nun sein Erstaunen darüber, dass dieses, Glas flach sei, wie ein Reiseglas;, er habe doch gedacht, es sei rund. Patient vermag nicht die in der Perspection oval erscheinende Peripherie des Glasrandes als rund aufzufassen."

„Ein hölzerner Ofenschirm in gewöhnlicher Stellung wird vom Patienten richtig erkannt. Hierauf wird der Gegenstand auf einen Tisch so gelegt, dass das obere Fnde des Schirmes dem Patienten zugewendet ist. Patient erklärt: „Eine Säge, . . . eine Sense.".

Die Fähigkeit, zu der Sehform die wirkliche Form zu associren, ist bei solchen Kranken wohl kaum je vollständig erloschen, meist 
Der Wille u. das räumliche Moment in Wahrnehmung u. Vorstellung. 313

handelt es sich nur um eine Abschwächung dieser Fähigkeit. Man kann beobachten, dass hin und wieder die gewöhnliche Sehform, wie sie die Gegenstände in ihrer gewöhnlichen Stellung liefern, noch mit der wirklichen Form bewerthet wird. Dagegen werden die Objecte in ungewöhnlichen Stellungen nicht erkannt.

Ist diese Auffassung gewisser Formen der Seelenblindheit richtig, dann müssen Objecte, deren Sehformen mit den wirklichen Formen übereinstimmen, bei denen also der psychische Act der Association sich erübrigt, besser erkannt werden. Solche Objecte sind ebene Figuren, z. B. Buchstaben. In der That ist dieses Symptom im Lis sauer'schen Falle vorhanden. Ich selbst habe es in einem anderen Falle beobachtet.

Wenn nun der Sehact als solcher durchaus nicht erklärt, warum wir unter allen möglichen Sehformen eines Objectes eine einzige, die, welche mit seiner wirklichen Form identisch ist, derartig bevorzugen, dasś sie immer beim Anblick des Objectes uns bewusst wird, so erhebt sich die Frage, welche Erlebnisse, welche Sinneswahrnehmungen es sind, die zur allmählichen Verdrängung der Sehform durch die wirkliche Form führen.

Das sind die Erlebrisse, die Sinneswahrnehmungen, welche unsere Willkürbewegungen begleiten. Von ihnen, den Tastempfindungen in weitesten Sinne des Wortes, bleiben die Vorstellungen der wichtigen Objectform in dem Bewusstsein als Erinnerungsbilder haften und erwerben ihre Ueberwerthigkeit den Sehformen gegenüber.

Ehe ich aber auf die Begründung dieser Behauptung eingehe, möchte ich noch genauer ausführen, inwieweit der sinnlich gestützte Antheil des räumlichen Moments unserer optischen Wahrnehmung von der wirklich in unserem Bewusstsein auftauchenden Raumvorstellung sich unterscheidet.

Dass die wirkliche Form der Dinge auf Rechnung der associativen Componente allein zu setzen ist, ist schoñ erörtert worden. Aber noch eine andere, allen gesehenen Objecten nothwendig zukommende räumliche Eigenschaft, die der absoluten Grösse, kann unmöglich auf das Bewusstwerden des durch den Sebreiz allein ausgelösten stereopsychischen Processes zurückgeführt werden. Halte ich meine Hand etwa $25 \mathrm{~cm}$ von meinem Gesicht entfernt, so erscheint sie mir keineswegs doppelt so gross als in $50 \mathrm{~cm}$ Entfernung. Ich sehe sie in beiden Fällen in ihrer "wirklichen" Grösse. Den 
Grössen der Netzhautbilder gemäss dürfte das nicht der Fall sein. Vollends erscheint mir ein Mensch in $20 \mathrm{~m}$ Entfernung auf der gegenüberliegenden Seite der Strasse nicht ebenso gross wie ein Maikäfer, der etwa $30 \mathrm{~cm}$ weit von meinem Gesicht vorüberfliegt; und doch sind die Netzhautbilder und damit die "Sehgrössen" beider Objecte gleich gross.

Betrachte ich das Zifferblatt der Wanduhr in meinem Zimmer, wenn ich dicht davorstehe, und trete dann $5 \mathrm{~m}$ bis an die gegenüberliegende Wand zurück, so bemerke ich durchaus keine Grössenabuahme des Zifferblattes, und doch bedeckt nunmehr das Netzhautbild nur noch den 25. Theil der Fläche.

Daraus folgt, die Vorstellung der wirklichen Grösse eines Objects, welche unweigerlich bei seinem Anblick in mein Bewusstsein tritt, ist keine directe Function des durch den Sehreiz erzeugten stereopsychischen Processes oder des Netzhautbildes. Den Erwachsenen auf der Gegenseite der Strasse sehe ich wirklich grösser als das Kind, das wenige Schritte vor mir hergeht, obgleich das Netzhautbild des letzteren das grössere ist.

Man könnte einwenden; dass die wahrgenommene Entfernung erst in Verbindung mit der Grösse des Netzhautbildes die Vorstellung der wirklichen Grösse bedingt, so dass die wirkliche Grösse des Objectes gleich $a \cdot d$ ist, wenn $a$ die Grösse des Netzhautbildes, d die wirkliche Entfernung bedeutet. Das ist zweifellos richtig, nur ist der Sehact an sich ebensowenig fähig, uns die Verstellung einer wirklichen Entfernung zu geben, wie die einer wirklichen Objectgrösse.

Bedenken wir, dass beim zweiäugigen Sehen eine Querdisparation von $5^{\prime \prime}$ in deutlicher Sehweite etwa $0,1 \mathrm{~mm}$ wirkliche Tiefenerstreckung bedeutet, in $1 \mathrm{~km}$ Entfernung aber einen Kilometer, so verstehen wir, dass in der That das unmittelbare räumliche Moment der optischen Wahrnehmung überhaupt nicht geeignet ist, die Vorstellung der absoluten Grösse hervorzubringen. Dazu fehlt das unveränderliche Vergleichsmoment. Nur wenn unter gleichen Verhältnissen die Reizung derselben zwei Sinneselemente stets dieselbe Grössenvorstellung in mir erweckt, wie es nicht bei der Netzhaut, wohl aber auf der Haut unseres Körpers der Fall ist, nur dann könnte diese Vorstellung gebildet werden.

Man kann sich in der That durch das Experiment unsehwer davon überzeugen, dass wir, auf unsere Sehwahrnehmung allein ange- 
Der Wille $u$. das räumliche Moment in Wahrnehmung u. Vorstellung. 315 wiesen, weder die Vorstellung der absoluten Entfernung noch die der obsoluten Grösse bilden könnten. Trifft man im Dunkelzimmer eine Vorrichtung derart, dass man auf einem beweglichen Papierschirm mit einem Scheinwerfer helle Kreise von beliebiger Grösse beleuchten kann, so hat der Beschauer, welcher ein- oder zweiäugig durch ein Loch in der Thüre beobachtet, keinerlei Wissen, weder von der Entfernung noch von der Grösse des beleuchteten Kreises. Wird der Versuch mit allen Vorsichtsmaassregeln angestellt, so ist zwar der Beobachter gezwungen, dem Kreise irgend eine absolute Grösse und Entfernung zu geben; denn eine Sehwahrnehmung ohne associative Componente gibt es nicht. Diese Vorstellung entspricht aber durchaus nicht der Wirklichkeit. Ein kleiner, ganz naher Kreis kann für sehr gross und zugleich sehr fern gehalten werden, ein grosser, sehr entfernter für sehr klein und ganz nahe. Es ist interessant, zu beobachten, wie in dem Augenblicke, wo ein bekanntes Vergleichsobject vorhanden ist, wenn z. B. der Experimentator seine Hand auf den Sehirm legt, im letzteren Falle ganz plötzlich der Kreis für ungeheuer gross gehalten wird ${ }^{\mathbf{1}}$.)

1) Es ist recht auffällig, dass die vielen Arbeiten, welche sich mit der scheinbaren Grösse der Gestirne am Horizont und im Zenith beschäftigen, diese Frage beantworten wollen, bevor die wichtige und unumgängliche Vorfrage erledigt ist, warum wir denn dem Monde z. B. überhaupt eine absolute Grösse beilegen. Die Versuche, die scheinbare Gestalt des Himmelsgewölbes, das eine Kugelcalote von geringer Höhe darstellen soll, dafür verantwortlich zu machen, sind natürlich gänzlich verfehlt. Denn auch diese scheinbare Wölbung ist nicht eine vom Sehact unmittelbar gelieferte Raumvorstellung, sondern ein associatives Moment.

Dass der Mond am Horizont sehr weit entfernt ist, das haben wir bei unseren Spaziergängen so und so oft sinnlich erfahren, oder wir haben den Mond doch immer nur hinter, d. h. gedeckt von den weitesten Objecten am Himmel aufsteigen sehen. Gemäss dieser sinnlich wahrgenommenen, beim Sebact associativ auftauchenden sehr grossen Entfernungsvorstellung bemessen wir die absolute Grösse des aufgehenden Mondes individuell verschieden, wie ein Scheunenthor, ein Wagenrad, einen Teller.

Von anderen als horizontalen Entfernungen aber haben wir keine sinnliche Erfahrung. Wir können uns aus eigener Kraft nicht senkrecht in der Luft erheben, und darum haben wir auch keine Veranlassung, in verticaler Richtung gesehene Objecte in die allergrösste durch Sinneserfahrung wahrnehmbare Entfernung zu verlegen.

Man ist immer wieder von Neuem erstaunt, wenn man gelegentlich das Zifferblatt einer Thurmuhr oder die Kugel auf dem Thurm aus unmittelbarer Nähe sieht, wie sehr man ihre wirkliche Grösse unterschätzt hatte. 
Also auch die Vorstellung der absoluten Grösse ist ein mittelbarer Antheil des räumlichen Momentes der Sehwahrnehmung. Sie ist als solche nicht gesetzmässig bestimmt, nicht die bewusste psychische Begleiterscheinung eines Mechanismus, der in der Netzhaut angreifeud eine durch unsere Organisation bestimmte gesetzmässige Veränderung im stereopsychischen Felde erzeugt, sondern sie ist abhängig von dem Zustande, in welchem das stereopsychische Feld durch alle voraufgegangenen Sinneserfahrungen sich gerade befindet. Die associative Componente der Sehwahrnehmung, die Vorstellung der wirklichen Form und Grösse, ist also eine Function der jeweiligen Erfahrung und mit dieser veränderlich.

Sie ist es allein, auf welcher alle sogenannten optischen Täuschungen beruhen, soweit sie sich auf räumliche Verhältnisse beziehen. In dem Maasse, wie ich die wirkliche Form dieser Täuschungsfiguren kennen lerne, in dem Maasse verschwindet - bei den einfacheren wenigstens - auch die Täuschung.

Diese Täuschungen dürften also bei Sehendgewordenen, frühzeitig Erblindeten und bei der geschilderten Form der Seelenblinden nicht auftreten. Leider bin ich bis jetzt nicht in der Lage gewesen, hierüber Untersuchungen anzutellen. Diese Täuschungen aber in Urtheils- und Wahrnehmungstäuschungen trennen $\mathrm{zu}$ wollen, ist ein betrübendes Zeugniss für den heutigen Stand der psychologischen Forschung, wie der grösste Theil der psychologischen Literatur über optische Täuschungen überhaupt.

Nur ganz kurz möchte ich an dieser Stelle auf die Entstehung dieser Täuschungen eingehen, die sich aus dem Vorhergehenden eigentlich von selbst ergibt.

Diese sinnliche Erfahrung über die wirkliche Grösse und Entfernung der Dinge führt also dazu, dass wir selbst kleine Netzhautbilder mit einer beträchtlichen absoluten Grösse bewerthen müssen; fehlt sie, so tritt diese Nöthigung nicht ein. Jedes Mal, wenn wir gehen, erfahren wir, dass die weiter entfernten Wegstrecken, deren Netzhautbild sehr klein ist, in Wirklichkeit viel grösser sind als die nächsten $10-20 \mathrm{~m}$, deren Netzhautbild ein grosses ist. Diese sinnliche Erfahrung machen wir nur in horizontaler Richtung und bewerthen danach Objectgrösse und Entfernung. Nie, oder doch nur in den seltensten Ausnahmefällen für verhältnissmässig kleine Erhebungen, machen wir ähnliche Erfahrungen in senkrechter Richtung, und desshalb schätzen wir senkrecht über einander gelegene Strecken von gleicher Grösse der Netzhautbilder für gleich gross, halten also alle Höhenerstreckungen für kleiner, als sie wirklich sind. 
Der Wille u. das räumliche Moment in Wabrnehmung u. Vorstellung. 317

Ein weisses Blatt Papier wird von mir als Ebene gesehen. Zeichne ich ein Sechseck darauf, so erregt dieses einfach den Eindruck einer ebenen Figur. (Fig. 1.) Vervollständige ich nun die
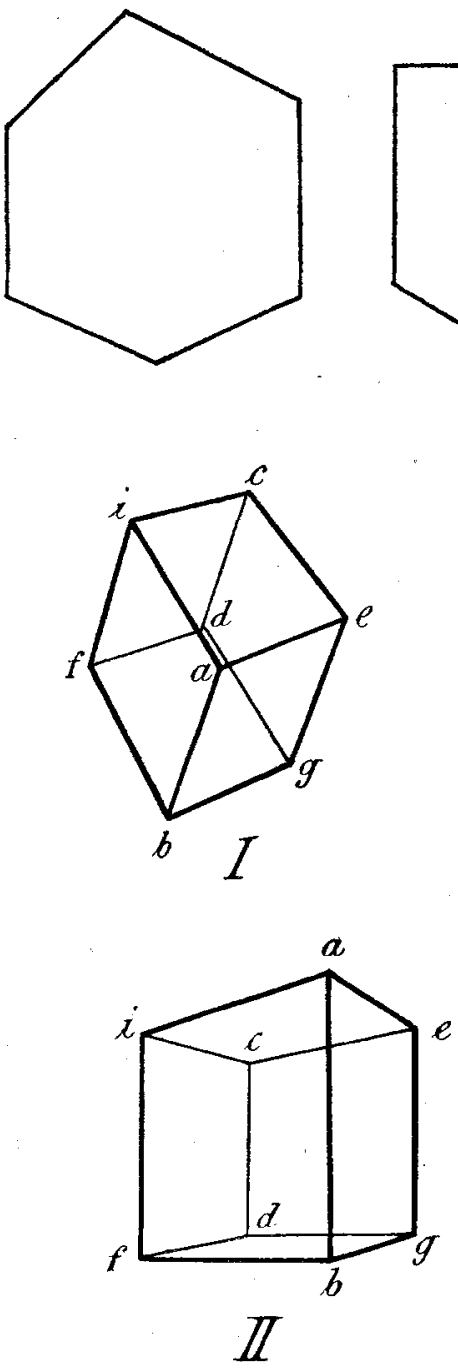

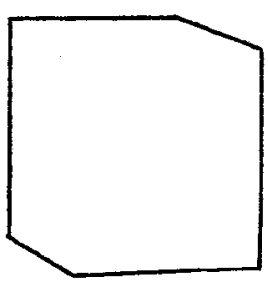

Fig. 1.
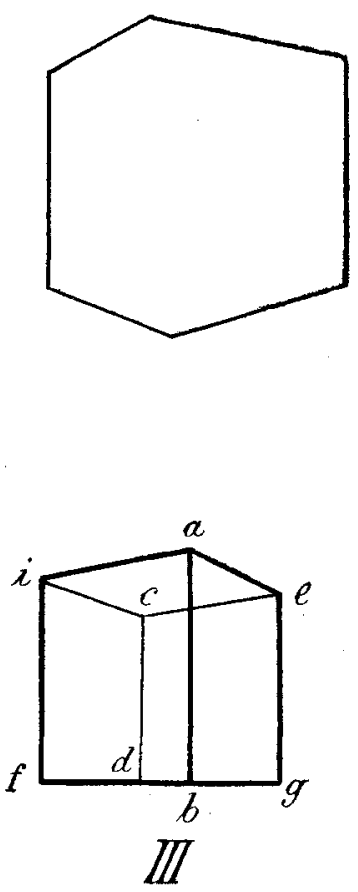

Fig. 2.

die Zeichnung etwa wie in Fig. 2 , so weiss ich zwar immer noch, dass ich eine ebene Zeichnung vor mir habe, aber ganz deutlich meldet sich zugleich in meinem Bewusstsein die Vorstellung des körperlichen Würfels. Ich weiss zwar genau, dass eine ebene Figur vorliegt, aber die Vorstellung des körperlichen Würfels tritt mit zwingender Gewalt in mein Bewusstsein, sie macht sich bei allen Grössen und Winkelschätzungen geltend.

Man beachte einmal, wie in Fig. 2, $I I I, a b$, als vordere Kante gedacht, bei unbefangener Betrachtung nicht wesentlich grösser erscheint als if, und wie Kante $i$ a fast borizontal verläuft! Und nun 
bemühe man sich, $c d$ als vordere Kante zu denken. Im Augenblicke, wo dies gelingt, erscheint die Neigung von $i$ a gegen die Horizontale sehr gross, $a b$ sehr bedeutend grösser als $i f$. Im ersteren Falle verhindert die associative Componente, zwar nicht in dem Maasse wie beim Erblicken eines wirklichen Würfels, aber noch deutlich genug, dass die verhältnissmässigen Grössen der auf der Netzhaut entstehenden Bilder von if und $a b$ zum Bewusstsein kommen; die associative Componente wirkt ausgleichend auf die der Sehform eigenen Grössenunterschiede. Im letzteren Falle bewirkt sie gerade das Gegentheil sie verstärkt dieselben.

Es war mir lehrreich, zu beobachten, dass bei Kindern von 6 bis 7 Jahren auch bei Zeichnungen die Sehform selbst so gut wie gar nicht bewusst wird. Ich stellte ihnen die Aufgabe, sich genau die Fläche $a$ e $c i$ in Fig. 2 anzusehen und liess sie dieselbe nachzeichnen. Eines dieser Kinder zeichnete ein Quadrat, alle aber zeichneten sie sehr quadratäbnlich, so dass man deutlich merkte, dass sie aus der Betrachtung nur die Erinnerung der wirklichen Form des gezeichneten Würfels zurückbehalten hatten. Sie liessen sich auch durchaus nicht durch den Augenschein von ihrem Irrthum überzeugen, und erst, als sie ihre Zeichnungen mit der Scheere ausgeschnitten hatten und versuchten, sie mit der Vorlage in Deckung zu bringen, gaben sie, sichtlich erstaunt, ibren Irrthum zu.

Die associative Componente macht sich also beim Sehen auch dann geltend, wenn, wie bei einfachen Strichzeichnungen, die Vorstellung des dreidimensionalen Gebildes ein Gegengewicht erhält in dem sicheren Wissen, dass ich eine ebene Zeichnung vor mir habe. Sie ist um so mächtiger, je genauer alle Einzelheiten der Beleuchtung, der Farbe der Wirklichkeit entsprechen, sie scheint bis zum Verschwinden abzunehmen, je unvollkommener die Zeichnung mit der Sehform bekannter Gegenstände übereinstimmt. Aber sie scheint eben nur zu verschwinden, sie verräth sich selbst dann noch in den Täuschungen über Grössen und Winkel, wenn ich mir der Anwesenheit einer körperlichen Vorstellung, die die Zeichnung erzeugen kann, gar nicht mehr bewusst bin.

Die mittelbare und die unmittelbare Raumcomponente beim Sehact zeigen also in ihrem gegenseitigen Bewusstseinswerth alle möglichen Abstufungen. Während ich beim Erblicken eines wirklichen Würfels nur rechte Winkel und gleich grosse Kanten sehe, fällt es mir beim Betrachten eines völlig naturgetreuen Gemäldes, 
Der Wille u. das räumliche Moment in Wahrnehmung u. Vorstellung. 319 einer einfacheren, schattirten Zeichnung, einer Strichzeichnung desselben Gegenstandes immer leichter, die Unterschiede der Kantengrössen, die Abweichungen der Winkel von $90^{\circ}$ in der Sehform wahrzunehmen. Die Sehform gewinnt in demeselben Maasse an Bewusstseinswerth, in welchem die Vorstellung des körperlichen Objectes zurücktritt.

In Fig. 3 findet man eine der beiden Müller-Ly er'schen Figuren in senkrechter Stellung. Kein Mensch dürfte hierbei etwas anderes zu sehen meinen als eine gerade Linie, an deren Enden je zwei Schräge angesetzt sind. Irgend eine körperliche Vorstellung

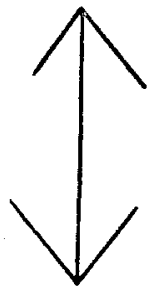

Fig. 3.

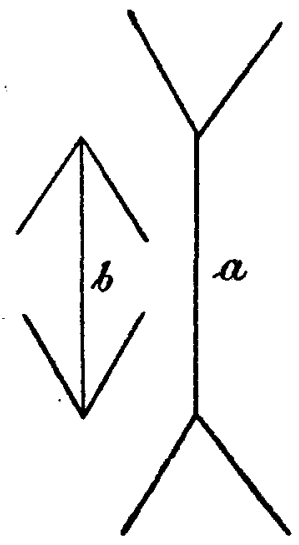

Fig. 4.

bemerken hierbei wohl nur die Wenigsten. In Fig. 4 sehen wir beide M üller-L y er' schen Figuren in senkrechter Stellung. Wir wissen, in beiden sind die Mittelstücke völlig gleich, und doch erscheint die Strecke in $4 a$ bedeutend grösser als in $4 b$. Jetzt betrachten wir nochmals Fig. $271.4 a$ ist derselbe Richtungscomplex, den wir an der Kante $c d$ sehen, während $4 b$ mit dem der Kante $a b$ übereinstimmt. Da wir die in der Zeichnung des Würfels bestehenden Unterschiede zwischen $a b$ und $c d$ zweifellos nur in Folge der sehr lebhaften associativen Componente zu übersehen geneigt sind, d. h. $c d$ für verbältnissmässig $\mathrm{zu}$ gross halten, so haben wir die MüllerL y er' sche Täuschung offenbar auch auf Rechnung dieser assoccativen Componente zu setzen. Denn in der Würfelzeichnung schätzen wir cd nur desshalb zu gross, weil wir wissen, dass es hinten liegt. Drehen wir die Verhältnisse in der Vorstellung um, denken wir $c d$ 
als vordere Kante, so unterschätzen wir seine Grösse. In der perspectivischen Würfelzeichnung aber sind es nur die Richtungen der mit $a b$ oben und unten zusammenstossenden Kanten, die spitzen Winkel, welche diese mit der Senkrechten bilden, die die Vorstellung des Vorne im Vergleich mit der Lage von $c d$ hervorrufen können, und dieser gleiche Unterschied findet sich in den Richtungscomplexen $4 a$ und $4 b$. Tritt bei Betrachtung dieser letzten Figuren nun auch die Vorstellung, dass bei $4 a$ das Mittelstück im Hintergrunde, bei $4 b$ vorne liegt, nicht deutlich auf, so zeigt sich der Einfluss dieser unbewussten associativen Componente doch in der Grössenschätzung ${ }^{1}$ ).

Dieses Anklingen der associativen Raumcomponente, ohne dass es zu einer klar bewussten körperlichen Vorstellung käme, ist nun

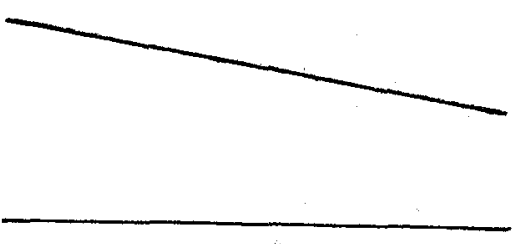

Fig. 5 .

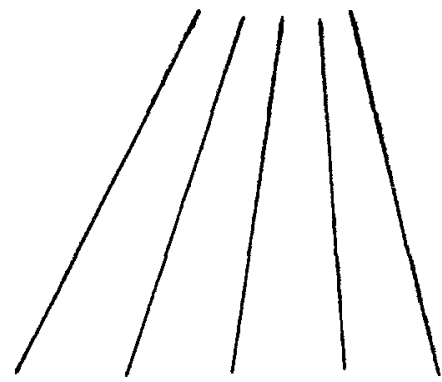

Fig. 6.

regelmässig die Ursache für das Auftreten von optischen Täuschungen der beschriebenen Art.

Zwei parallele Gerade geben immer die Sehform zweier sich schneidenden Linien. Entsteht beim Betrachten zweier convergent gezeichneter Linien in unserem Bewusstsein diese Sehform (Fig. 5), so wirkt immer die associative Componente mit, deren Bewusstseinswerth dieVorstellung paralleler, vom Beschauer sich entfernender Geraden ist.

Erfordert es bei Betrachtung von Fig. 5 eine gewisse Mühe, diese associative Componente zur Vorstellung zu machen, so bemerken wir sie beim Anblick der Fig. $2(I, I I, I I I)$ an den Kanten $\alpha e$, $b g, a i, b f$ obne Weiteres, und zwar ist in Fig. $2, I$ diese Vorstellung der Parallelität so vorwiegend, dass wir die wirkliche, durchaus nicht unbedeutende Convergenz der Kanten nicht wahrnehmen und in Folge dessen $a b=i f=e g$ schätzen.

1) Gelingt es, das Mittelstück von $4 b$ als hinten liegend vorzustellen, so verschwindet die Täuschung. 
Der Wille u. das räumliche Moment in Wahrnehmung u. Vorstellung. 321

Die Fig. 6, erzeugt dieselbe Sehform wie die Dielenfugen eines Zimmerfussbodens. Während aber diese Sehform, wenn ich wirklich einen solchen Fussboden sehe, gar keinen Bewusstseinswerth hat, scheint umgekehrt die associative Componente beim Anblick von Fig. 6 belanglos. Freilich, wenn ich erst einmal darauf aufmerksam geworden bin, dass Fig. 6 die Dielenfugen darstellen kann, dann tritt auch die associative Componente als Vorstellung auf. Aber ein Unbefangener sieht in zwei aufeinander zulaufenden Geraden zunächst sicher nichts Räumliches. Und doch lässt sich auch bei ihm die Einwirkung der associativen Componente deutlich nachweisen.

Auf wirklichen Parallelen senkrechte Verbindungslinien (die Fugen eines mit rechteckigen Steinen belegten Bürgersteiges) haben

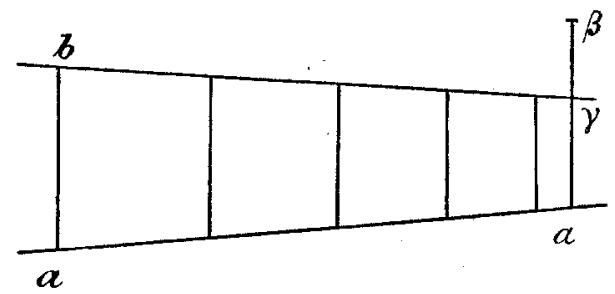

Fig. 7.

eine desto kleinere Sehgrösse, je weiter sie von uns entfernt sind. Trotzdem ruft ihr Anblick in uns, recht unabhängig von den colossalen Unterschieden der Sehgrössen, die Vorstellung der gleichen wirklichen Grössen hervor.

Dieser Zwang, der jedes Mal sich zeigt, wenn die Sehform zweier convergenter Linien in uns erregt wird beim Anblick eines räumlichen Objectes, besteht nun auch z. B. bei Anblick von Fig. 7 . $a b$, ist thatsächlich genau gleich $\alpha \beta$, und wenn wir auch sehen, dass die auf $a b$ nach rechts folgenden Verbindungslinien immer kleiner werden, so unterschätzen wir doch diese Grössenabnahme, ganz der Wirkung der associativen Compenente entsprechend; wir würden es desshalb kaum für möglich halten, dass $\alpha \gamma$ um $\beta \gamma$ kleiner ist als $a b$, und überschätzen $\alpha \beta$ bedeutend.

Ganz ähnlich, nur etwas stärker, tritt die Täuschung in Fig. 8 hervor, wo $\alpha \beta=\alpha^{\prime} \beta^{\prime}$ und $\gamma \delta=\gamma^{\prime} \delta^{\prime}$ ist; $\varepsilon \xi$ ist dabei gleich $\alpha \beta$, wird aber für grösser gehalten.

Die associative Componente bewirkt also, dass wir zwischen Convergirenden gelegene Strecken für um so grösser halten, je 
näher sie am Schnittpunkt liegen. Dabei hebt die associative Componente bisweilen eine deutliche Tiefenvorstellung in's Bewusstsein.

Die Täuschung in Fig. 9 ist danach ohne Weiteres verständlich, sowohl in $9 a$ wie in $9 b$ sind sämmtliche Vierecke einander gleich. In der Richtung der Convergenz der schrägen Seiten aber besteht eine deutliche Neigung zur Grössenüberschätzung.

Ja, die einfache Zeichnung eines schiefen Winkels verräth deutlich das Vorhandensein der associativen Componente. In der überwiegenden Mehrzahl der Fälle associren wir zu der Sehform

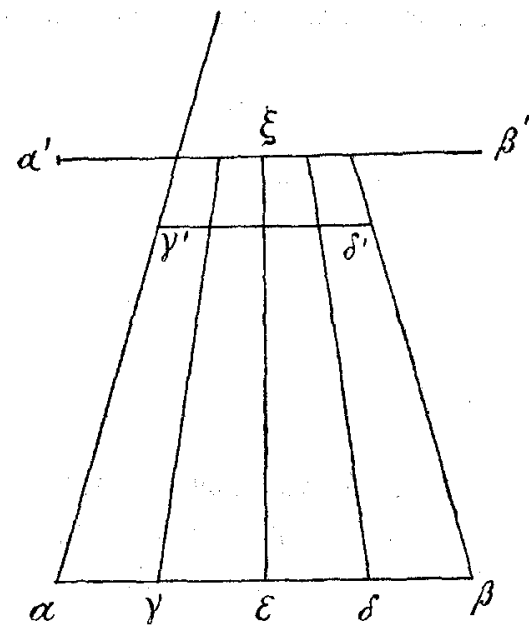

Fig. 8.
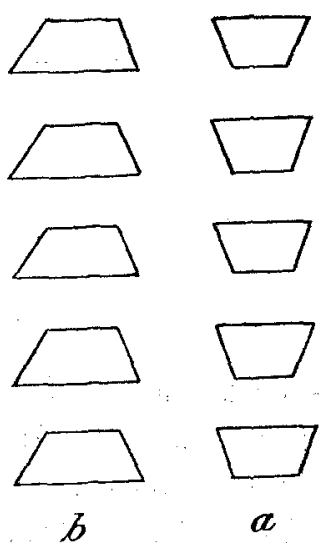

$a$

eines schiefen Winkels die wirkliche Form eines solchen von $90^{\circ}$. Diese Form bevorzugen wir aus angeborener Anlage, wie ich gezeigt habe, bei aller produktiven Thätigkeit unter allen möglichen Winkelformen. Sowohl also aus Gründen der Erfahrung wie aus Gründen der uns angeborenen Hirnorganisation tritt die Sehform des schiefen Winkels mit einer associativen Componente in Verbindung, die den Bewusstseinswerth eines rechten Winkels hat.

Nur die Sehform des rechten Winkels ist im Allgemeinen identisch mit dem Bewusstseinswerth des dazu associirten räumlichen Momentes. Hieraus erklärt sich folgende eigenthümliche $\mathrm{Er}$ scheinung. Einen Winkel von $45^{\circ}$ schätzen wir zu gross; zeichnen wir zwei Winkel von $45^{\circ}$ mit gemeinsamem Scheitel und Schenkel, 
Der Wille u. das räumliche Moment in Wahrnehmung u. Vorstellung. 323

so erscheint jeder einzelne grösser, als er wirklich ist, beide zusammen aber sehen durchaus so aus wie ein Rechter. Fig. 10.

Das ist natürlich nur unter Zuhülfenahme einer dreidimensionalen Formvorstellung verständlich, die sofort deutlich wird, wenn ich mir $A \approx$ senkrecht auf der Ebene des Papiers denke.

Ja, eine einfache, schräg zu den Rändern des Zeichenblattes gestelite Grade kann ich mir denken als das Bild eines Telegraphendrahtes, der in der Richtung der Senkung sich von mir entfernt, oder als oberen Rand einer Mauer, die senkrecht zur Ebene des Papiers steht. Die associative Componente ist mit der Sehform gegeben und kann in jedem Augenblicke Vorstellung werden.

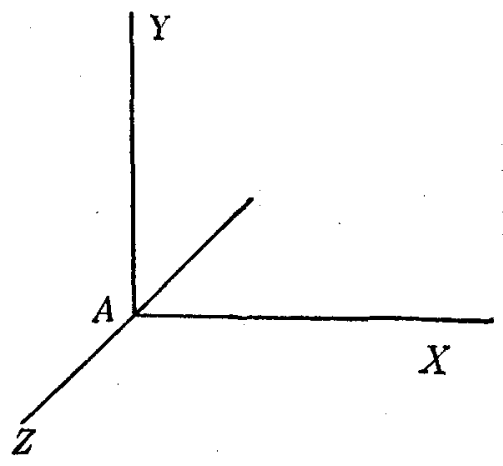

Fig. 10.

Aber sie wirkt mit, selbst wenn ihr kein Bewusstseinswerth zukommt. In Fig. 12 sind skizzenhaft zwei Häuser dargestellt, wie sie erscheinen, wenn ich eine Strasse entlang sehe. Das Haus II springt etwas mehr in die Strasse vor als Haus I. Diese ohne Weiteres auftauchende räumliche Vorstellung wird durch die schrägen Linien $c d$, ef u. s. w. erzeugt. Dort, wo sie unterbrochen sind, an den Senkrechten bei $a b$, deckt sich die associative Componente mit der Vorstellung der Zeichenebene; $\varepsilon \varphi$ ist die Verlängerung von $c d$. Diese Vorstellung aber kann nicht aufkommen, weil die hier als Vorstellung sehr deutliche associative Componente verlangt, dass $\varepsilon \varphi$ in einer anderen Ebene liegt als $c d$. Die Punkte $d$ und $\varepsilon$ liegen in einer Ebene $a b e d$. In dieser Ebene bedeutet aber $\varepsilon b$ eine wirklich kleinere Strecke als $a d$; daher liegt $\varepsilon$ tiefer als $d$. Hier verhindert also die associative Componente die einheitliche Auffassung von $c d \varepsilon \varphi$. 
E. Storch:

Man wird nun obne Weiteres die Täuschung der Poggen d orf'schen Fig. 12 begreifen. $\Lambda \mathrm{n}$ den Stellen der Zeichenebene, wo die Schrägen

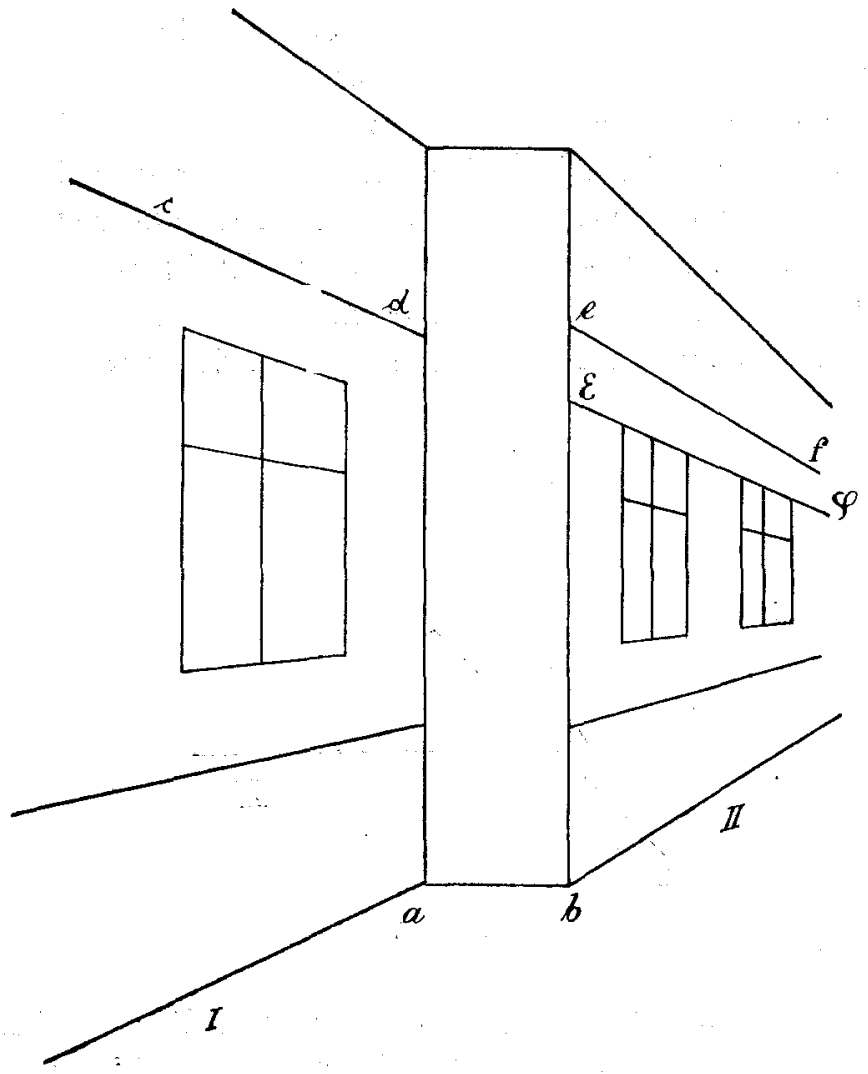

Fig. 11.

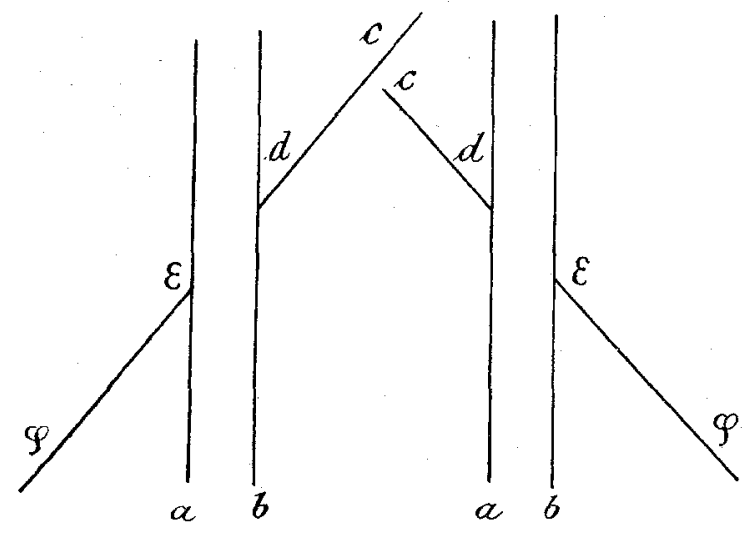

Fig. 12. 
Der Wille $u$. das räumliche Moment in Wahrnehmung u. Vorstellung. 325

$c d$ und $\varepsilon \varphi$ vorhanden sind, wirkt die associative Componente, die, zum klaren Bewusstsein erhober, dieselben räumlichen Vorstellungen schaffen würde, wie wir sie bei Fig. 11 bemerken. Nur zwischen den Parallelen $a \varepsilon$ und $b d$ ist das associative Moment mit dem sinnlich gestützten identisch. Darum erscheint $\varepsilon$ nicht in der Verlängerung von $c d$, sondern tiefer zu liegen.

Wohl verstanden: Ich behaupte nicht, dass für das Zustandekommen der Täuschung die entsprechende dreidimensionale Vorstellung im Bewusstsein vorhanden ist. Wohl aber behaupte ich, dass ein Hirnprocess, welcher der associativen Componente entspricht, und dessen Vorhandensein durch die bei jedem Sehact vorhandene Vorstellung der absoluten Grösse bewiesen ist, auch insofern er selbst keinen Bewusstseinswerth hat, den unmittelbaren Antheil der räumlichen Sehwahrnehmung bezw. den ihm zu Grunde liegenden stereopsychischen Process in deutlicher Weise beeinflusst, so beeinflusst, wie eben die Vorstellung der Sehform durch die bewusste Vorstellung der wirklichen Form beeinflusst werden müsste.

Genau genommen sind es bei diesen Täuschungsfiguren aber eigentlich 2 associative Componenten verschiedener Herkunft, die um den Vorrang im Bewusstsein kämpfen. Dass ich beim Anblick eines wirklichen Würfels gleiche Kanten und rechte Winkel sehe, während der Richtungscomplex der Sehform keinen deutlichen Bewusstseinswerth hat, dass ich aber beim Anblick einer Würfelzeichnung neben der Vorstellung der wirklichen Form auch die Sehform im Bewusstsein habe, dieser Unterschied der räumlichen Vorstellungen bei gleichen Sehformen kann ja nur darauf beruhen, dass ich die wirkliche Form des Papierbogens kenne, dass mir der von dem Papier ausgeübte Netzhautreiz als associative Componente die wirkliche Form und Grösse des Blattes in's Bewusstsein hebt.

Dass diese letztere associative Componente es ist, welche das Bewusstwerden der reinen Sehform begünstigt, wird dadurch bewiesen, dass, wenn ich diese Figuren auf einen unsichtbaren Hintergrund, z. B. auf eine Glasplatte, zeichne, die zugehörigen drei dimensionalen Vorstellungen viel deutlicher sich entwickeln. Einen aus Draht hergestellten Würfel vermag ich von seiner aus Draht bergestellten perspectivischen Zeichnung bei einäugiger Betrachtung überhaupt nicht zu unterscheiden. Ist seine Kantenlänge $5 \mathrm{~cm}$, so 
genügt eine Entfernung von $10 \mathrm{~m}$ und weniger, um die Unterscheidung unmöglich zu machen für zweiäugiges Sehen.

Ohne hier auf die Unzahl aller bekannten Täuschungsfiguren einzugehen, möchte ich nur bemerken, dass nicht eine einzige existirt, welche durch die associative Componente nicht zwanglos erklärt würde.

In dem Zusammenwirken der beiden Antheile des räumlichen Momentes beim Sehen liegt ohne Frage eine Erscheinung vor, die den Namen Association verdient.

Aber wiederum bemerken wir, dass die hier stattfindende Association durchaus nicht eine einfache Addition von Bewusstseinserscheinungen ist. Sehe ich nach einer Zimmerecke hin, so ist lediglich die associative Componente im Bewusstsein vertreten, ich erhalte durch den Sinnesreiz nur die Vorstellung dreier unter rechten Winkeln zusammenstossender Ebenen. Die unmittelbare Componente ist hierbei keine Vorstellung.

In der einfachen Strichzeichnung des Würfels ist das Verhältniss beider Componenten ein anderes. Ich bemerke viel besser als beim Anblick des wirklichen Würfels, dass die gezeichneten Kanten nicht gleich gross, die gezeichneten Winkel keine rechten sind; aber trotzdem ist die associative Componente als räumliche Würfelvorstellung deutlich vorhanden, und bewirkt, dass ich die Grössenunterschiede in den gezeichneten Kanten, ebenso die Abweichung der Winkel von $90^{\circ}$ mehr weniger unterschätze,

Endlich in den sogenannten optischen Täuschungsfiguren tritt die associative Componente an Bewusstseinswerth fast vollständig. zurück, so dass ich mir einer Körpervorstellung meist gar nicht bewusst bin. Immerhin ist es durch einen Act der Aufmerksamkeit viel leichter, diese Körpervorstellung in's Bewusstsein zu heben, als beim Anblick einer Zimmerecke die unmittelbare Componente Vorstellung werden zu lassen.

Das Vorhandensein des Hirnprocesses, welcher dieser associativen Componente zu Grunde liegt, verräth sich aber sehr deutlich darin, dass wir bei den Täuschungsfiguren Richtungen und Grössen in demselben Sinne falsch beurtheilen, wie wenn die gleichen Netzhautbilder durch wirkliche körperliche Objecte erzeugt werden.

Die Association dieser beiden räumlichen Componenten besteht demnach nicht in einer einfachen Addition, sondern in einer gegenseitigen Veränderung oder Beeinflussung, in der Bildung einer Ueber- 
gangsvorstellung, in welcher bald die eine, bald die andere Componente überwiegen kann.

Würden die Psychologen die zwei Bestandtheile im räumlichen Momente jeder Sehwahrnehmung erkannt haben, würden sie die Unvorstellbärkeit der von jeder Erfahrung unabhängigen Componente und damit die Unmöglichkeit, die Sehwahrnehmung ohne Zusammenhang mit anderen Sinneswahrnehmungen zu behandeln, begriffen haben, ein grosser Theil der Arbeiten über optische Täuschungen wäre ungeschrieben geblieben; kaum zum Schaden der Wissenschaft.

Das eigenthümliche Verhalten, welches die Sehform gegenüber der wirklichen Form der Objecte in unserem Bewusstsein zeigt, verdient noch eine besondere Beleuchtung. Wir können durch einen besonderen Akt der Aufmerksamkeit, z. B. beim Körperzeichnen, die Sehform voll in's Bewusstsein heben. Obne dass der Sinnesreiz ein anderer wäre, $d . h$. bei genau den gleichen nervösen Processen in der Netzhaut und den subcorticalen Endigungsstätten des Sehnerven, finden wir bei unbefangener Betrachtung in unserem Bewusstsein einen ganz anderen Richtungscomplex : die wirkliche Form. Der stereopsychische Vorgang, welcher der unmittelbaren Raumcomponente der Sehwahrnehmung entspricht, hat also eigentlich keine subjective Seite, aber er ist die nächste mechanische Ursache für einen anderen stereopsychischen Process, den wir als Vorstellung der wirklichen Objectform erleben.

Meiner Ansicht nach würde man sich gröblich an dem Wortbegriffe "Vorstellung" versündigen, wollte man hier die un mittelbare Componente der Sehwahrnehmung als unbewusste Vorstellung bezeichnen. Theile dieser Componente können in's Bewusstsein treten: die sind dann Vorstellung. Diejenigen Theile aber, welche keine bewusste Begleiterscheinung haben, sind nichts als chemische Processe in der Nervensubstanz, die in der Kette der mechanischen Causalität keine andere Rolle spielen als die Veränderung der Netzhaut durch Lichtreize oder die äussere Lichtenergie.

Dass wir im Stande sind, auf Grund rein psychologiseher Betrachtungen die nächsten mechanischen Ursachen der Veränderungen unseres Bewusstseinsorganes zu erschliessen, das eben stellt den Werth der Psychologie für unsere mechanische Weltauffassung erst in's rechte Licht.

Die Psychologie greift in dieser Kette der mechanischen Causa- 
lität gerade im Mittelpunkte an, während Physik und Physiologie von aussen her diesem Mittelpunkte zustreben. Sie ist die Wissenschaft von innen heraus, die einst der Wissenschaft von aussen die Hand reichen wird.

Unsere Betrachtungen über das räumliche Moment in Wahrnehmung und Vorstellung wären unvollständig; wollten wir es nicht auch in seiner Rolle bei den Willkürbewegungen untersuchen. Gerade die Beziehungen zwischen Raumvorstellung und Willkürbewegung gestatten ein Eindringen in ungeahnte Tiefen des Mechanismus unserer Bewusstseinserscheinungen.

Für jede willkürliche Muskelinnervation ist es unbedingtes Erforderniss, dass ihr in unserem Bewusstsein eine Vorstellung vorausgeht, welche die erfolgende Bewegung gesetzmässig bestimmt. Diese Vorstellung ist entweder eine räumliche oder eine lautliche. Im letzteren Falle kommt für die Ausführung immer nur eine engbegrenzte Gruppe von Muskeln in Betracht: der phonetische Apparat. Will ich den Buchstaben A oder B aussprechen, oder auch ein Wort, z. B. Haparanda, so kann ich das immer nur durch die Innervation ganz bestimmter Muskeln, und diese Innervation ist durch die Lautvorstellung in allen ihren Einzelheiten genau bestimmt.

Ist aber die den Bewegungswillen begleitende Vorstellung eine räumliche, z. B. die eines Kreises, einer geraden Linie, der Buchstabenform A oder B, so vermag ich die Bewegung durch sehr verschiedene Muskelgruppen auszuführen. Ich kann den Kreis mit dem rechten oder linken Arm, mit einem beliebigen Finger allein, mit dem Kopf, ja, schliesslich mit jedem genügend beweglichen Punkte meines Körpers beschreiben. Ich kann diesen Weg auch durch eine beliebige Fortbewegungsart meines ganzen Körpers durchlaufen, iç kann ihn gehen, kriechen, schwimmen, fahren u. s. w.

Die einfache Formvorstellung ist also der Lautvorstellung in Bezug auf die Muskulatur unseres Körpers nicht gleichwerthig. Während die Lautvorstellung die bewusste Begleiterscheinung eicer Hirnrindenveränderung ist, die nur für die motorischen Rindenfoci des phonetischen Apparates einen adäquaten Reiz bildet, kann der stereopsychische Process, den wir als Vorstellung einer Form erleben, je nach den Umständen zum adäquaten Reiz für die motorischen Foci fast aller Körpermuskeln werden.

Diese Willkürbewegungen, welche von Raumvorstellungen abhängig sind, bezeichne ich als räumliche Bewegungen. Sie sind es, 
welche uns in Folgendem ausschliesslich beschäftigen werden. Die Formvorstellung eines Kreises ist nun nicht ohne Weiteres identisch mit der Vorstellung der Kreisbewegung. Während bei ersterer der ganze Kreis in allen seinen Theilen gleichwerthig im Bewusstsein vorhanden ist, schliesst sich bei letzterer an ein ganz besonders beachtetes Kreisdifferential im nächsten Zeittheilchen das räumlich benachbarte an u. s. f., bis die Aufmerksamkeit nach einer gewissen Zeit bei dem Ausgangspunkte wieder anlangt. Die Bewegungsvorstellung entsteht also aus der Formvorstellung dadurch, dass die räumlich benachbarten Wegdifferentiale in den aufeinanderfolgenden Zeittheilchen besonders beachtet werden, d. h. dadurch, dass in jedem Augenblicke der Bewegungsvorstellung ein ganz bestimmter Wegdifferential einen besonderen Bewusstseinswerth erhält, den in diesem Zeittheilchen die übrigen Wegdifferentiale nicht besitzen.

Entsteht die Bewegungsvorstellung in Folge eines sinnlichen Reizes, sehe ich z. B. eine Kegelkugel rollen, oder schreibt mir Jemand einen Buchstaben mit der Fingerspitze auf die Haut der Hand oder des Rückens, so vermag ich genau zu sagen, welches der Grund für dieses Wandern der Aufmerksamkeit ist. Der wechselnde Sinnesreiz erzeugt durch Vermittlung der Sinnescentren in jedem Augenblicke eine besondere, sinnlich gestützte, stereopsychische Erregung, und diese bedeutet für unser Bewusstsein die mit Aufmerksamkeit betrachtete Vorstellung eines ganz bestimmten Punktes im Raum.

Kann ich mir einen bestimmten Punkt im Raume vorstellen, oder meine Aufmerksamkeit unverändert auf einen bestimmten Raumpunkt lenken, wofern derselbe nicht räumliches Moment einer Sinneswahrnehmung ist? Mit anderen Worten: Gibt es eine Aufmerksamkeit auf einen Raumpunkt, falls dieser nicht sinnlich wahrgenommen wird? Berühre ich eine auf dem Tische liegende Schrotkugel bei geschlossenen Augen mit der Zeigefingerspitze, so ist das räumliche Moment der Wahrnehmung ein ganz bestimmter Punkt im Raum.

In dem Augenblicke, wo ich die Fingerspitze zurückziehe, wird der Punktvorstellung die sirnliche Stütze entzogen. Die Wahrnehmung wird zur Vorstellung im landläufigen Sinne des Wortes oder zum Erinnerungsbilde. Behalte ich die Augen geschlossen, so kann ich nunmehr auf Grund dieser Vorstellung die Kugel nochmals mit dem Zeigefinger berühren wollen. Die Raumvorstellung bezw. der ihr zu Grunde liegende stereopsychische Process wird zur Vorstufe einer motorischen Innervation. Der Versuch aber zeigt, dass die Vor- 
stellung des Raumpunktes nicht mehr identisch ist mit dem räumlichen Moment der Tastwahrnehmung. Ich treffe die Schrotkugel nicht, was sicher mit jedem beliebigen Körpertheile gelingt, solange ich die Kugel fühle. Der stereopsychische Process ist also ein anderer geworden, denn sonst müsste er sich in der gleichen Muskelinnervation entladen wie während der Wahrnehmung. Diese Veränderung verräth sich meinem Bewusstsein als ein Gefühl der Unbestimmtheit.

Wir sahen, dass beim einäugigen Sehact die Anordnung der Sehpunkte in der Tiefenausdehnung nicht sinnlich gestützt ist. Die Entfernung eines einäugig wahrgenommen Punktes ist demnach eine blosse, sinnlich nicht gestützte Vorstellung.

Betrachten wir mit beiden Augen eine senkrecht und frei stehende Stricknadel, und machen den Versuch, diese mit einer horizontal von der Seite her eingeführten Sonde zu berühren, so gelingt dieser Versuch ohne Weiteres. Wir nehmen in jedem Augenblicke sehr deutlich war, ob sich der Sondenknopf in einer näheren oder ferneren Ebene befindet als die Stricknadel. Schliessen wir ein Auge, so fahren wir vorne oder hinten an der Stricknadel vorbei. Das räumliche Moment des Bewegungswillens, die stereopsychische Vorstufe der Muskelinnervation ist eine andere geworden. Die Entfernung in der Tiefenrichtung ist nunmehr blosse Vorstellung, nicht mehr sinnlich gestützt.

Da nun das Wesen der Bewegungsvorstellung darin besteht, dass die Aufmerksamkeit in zeitlich benachbarten Theilchen sich auf immer andere Raumpunkte richtet, so ist die Möglichkeit, eine bestimmte Willkürbewegung auszuführen, lediglich davon abhängig, ob ich in jedem Augenblicke der Bewegung eine sinnlich gestützte Vorstellung von dem Orte des zu bewegenden Körpertheiles habe. Denn nur eine solche sinnlich gestützte Raumvorstellung vermag, wie die Beispiele zeigen, Vorstufe einer ganz bestimmten Innervation zu werden.

D. h. Vorbedingung für jede Willkürbewegung ist, dass wir den Ort des zu bewegenden Körpertheiles sinnlich wahrnehmen.

Das ist nun unter physiologisehen Verhältnissen immer der Fall. Wir können unseren Körper sehen und bei geschlossenen Augen auch fühlen, so dass wir mit grosser Sicherheit die Nasenspitze, den Ellenbogen, jedes beliebige Fingergelenk berühren können. Denn fortwährend finden an den Stellen unseres Körpers, die sich berühren, ferner' durch den Druck der Kleidung, durch die Luftströmung, durch 
Der Wille u. das räumliche Moment in. Wahrnehmung u. Vorstellung. 331

kleine, unwillkürliche Bewegungen Reizungen der Tastnerven und der tiefen Sensibilität statt, und diese Sinnesempfindungen müssen ein räumlicbes Moment besitzen.

Nun ist es obne Weiteres ersichtlich, dass wohl niemals bei einem Menschen alle Einzelheiten dieser stereopsychischen Erregung. als deutliche Formvorstellung des Körpers im Bewusstsein vorhanden sind. Aber wie ich den unmittelbaren Antheil des räumlichen Momentes beim Sehen durch eine Anstrengung der Aufmerksamkeit in's Bewusstsein zu heben vermag, so kann ich auch jede beliebige Stelle meines Körpers durch einen Aufmerksamkeitsact in all ihren räumlichen Beziehungen zur klar bewussten Vorstellung werden lassen. Ich habe dann die eindeutig bestimmte, sinnlich gestützte Vorstellung des Ortes, wo sich der betreffende Körpertheil z. Z. befindet.

Wiederum bedeutet die Richtung der Aufmerksamkeit auf einen bestimmten Körpertheil offenbar eine Veränderung des stereopsychischen Vorganges, der dieser Bewusstseinserscheinung zu Grunde liegt. Denn nunmehr erst ist dieser stereopsychische Vorgang geeignet, Vorstufe der Willkürbewegungen dieses Körpertheiles zu werden.

Es ist vielleicht nicht überflüssig, diese theoretisch abgeleiteten Vorbedingungen der Willkürbewegungen an unseren klinischen Erfahrungen zu prüfen.

Wir kennen von den Rückenmarks- und Gehirnkrankheiten her die Erscheinung, dass die Kranken die Lage ihrer Glieder nicht mehr mit der nöthigen Genauigkeit wahrnehmen. Die Fähigkeit, bei geschlossenen Augen einen bestimmten Körpertheil zu berühren, z. B. die beiden Zeigefingerspitzen in Berührung zu bringen, hat dann mehr weniger gelitten, die Kranken verhalten sich ähnlich wie Gesunde beim Versuch, nach Schluss eines Auges von der Seite her mit der Sonde die Stricknadel zu treffen oder eine Nadel einzufädeln. Das räumliche Moment der Körperwahrnehmung hat die sinnliche Stütze zum Theil verloren. Gibt man diesem räumlichen Moment die nöthige sinnliche Stütze, welche zur Ausführung der Bewegung nöthig ist, lässt man den Kranken die Augen öffnen, so erfolgt die Bewegung mit viel grösserer Sicherheit.

In Fällen aber, wo die Sensibilität eines Gliedes ganz erloschen ist, pflegt bei Ausschluss des Sehvermögens die Fähigkeit zu Willkür- 
bewegungen überhaupt zu erlöschen. G. B. Duchenne ${ }^{1}$ ) bringt hierfür eine Reihe von Beobachtungen bei: Er stellte fest, „dass gewisse Individuen, sobald man sie am Sehen verhindert, die Fähigkeit verlieren, ihre willkürlichen Bewegungen auszuführen. Wenn man ihnen dabei z. B. befiehlt, die Hand zu schliessen oder zu öffnen, den Vorderarm zu beugen oder $\mathrm{zu}$ strecken, mit einem Worte, bei jeder Bewegung, zu der man sie auffordert, verharren die Muskeln trotz aller ihrer Bemühungen in vollkommener Unthätigkeit." . . A All diese Bewegungen konnten sie unter Mitwirkung des Gesichtsinnes ausführen.

Wenn wir nun weiter erwägen, dass keineswegs ein gesetzmässiger Zusammenhang zwisehen Gefühlsstörungen einerseits und Störungen der Willkürbewegungen andererseits besteht, derart etwa, dass man aus ersterer diese letztere vorausbestimmen könnte, so müssen wir die Bedeutung der Sensibilität für die Ausführung unserer Willkürbewegungen lediglich in dem Umstande sehen, dass sie uns mit einem gewissen nothwendigen Grade der Bestimmtheit eine răumliche Vorstellung von dem zu bewegenden Körpertheile gibt.

Irgend ein Object können wir nur in einer gewollten Richtung bewegen, wenn wir den Ort, an dem er sich befindet, mit hinrejchender Genauigkeit kennen; das gilt von der auf dem Tische liegenden Schrotkugel ebenso wie von unseren Gliedmaassen. Dieser nothwendige Grad von Genanigkeit ist aber nur vorhanden, solange das räumliche Moment eine ausreichende sinnliche Stütze hat.

Welche Komponenten der Sensibilität hierfür von besonderer Bedeutung sind, ist z. Z. noch nicht sicher. Es scheint klinischen Erfahrungen zu Folge, als ob besonders die Elemente der tiefen Sensibilität, diejenigen Nervenapparate, für welche die Körperbewegungen einen Sinnesreiz abgeben, eine besondere Rolle spielen. Wenigstens glaubt $\mathrm{O}$. Förster ${ }^{2}$ ), aus seinen Beobachtungen diesen Schluss ziehen zu müssen.

Ich bin der Ansicht, doch auch diese Frage zum grossen Theil erst durch psychologische Analyse der experimentellen Forschung zugänglich gemacht werden muss. In meinem Versuch einer psychophysiologischen Darstellung der Sinneswahrnehmungen ${ }^{3}$ ) habe ich die

1) Physiologie der Bewegungen S. 614. Cassel und Berlin 1885 .

2) Untersuchungen üher das Localisationsvermögen bei Sensibilitätsstörungen. Monatsschr. f. Psychiatrie und Neurologie Bd. 9 S. 31 und 131.

3) S. Karger, Berlin 1902, Cap. 9. 
Der Wille u. das räumliche Moment in Wahrnehmung u. Vorstellung. 333

Rolle, welche die verschiedenen beim Tasten in Betracht kommenden Sinnesqualitäten für das Zustandekommen der Raumvorstellung spielen, einer Untersuchung unterzogen, und gelangte zu der Anschauung, dass weder die in der Haut gelegenen Sinneselemente, welche die Berührungsempfindung vermitteln, noch die der tiefen Sensibilität, die bei Bewegungen gereizt werden, für sich allein das räumliche Moment erzeugen können. Erst das Zusammenwirken beider vermag uns die dreidimensionale Vorstellung eines Raumpunktes eindäutig zu geben. Der Hautsinn als solcher ordnet ähnlich wie der einäugige Sehact die Raumpunkte nur zweidimensional an, und erst in Verbindung mit der an sich ebenfalls rudimentären Raumcomponente der tiefen Sensibilität, entsteht ähnlich wie beim zweiäugigen Sehen die aus drei psychischen Elementen zusammengesetzte Raumvorstellung des Tastobjects ${ }^{1}$ ).

Allein die Frage, welche Sinneselemente der Raumvorstellung eines Körpertheiles diejenige sinnliche Stütze geben, welche zur Ausführung einer Willkürbewegung nothwendig ist, hat hier kein wèsentliches Interesse. Die "Hauptsache ist, dass diese Raumvorstellung in jedem Augenblicke der Bewegung mit der nöthigen Genauigkeit vorhanden ist. Für den stereopsychischen Process selbst, den wir als Ortsvorstellung erleben, ist es ja gleich, auf welche Weise er zu Stande kommt. So lange wir in den Raumvorstellungen Verschiedenheiten nicht feststellen können, liegt ihnen der gleiche Hirnprocess im stereopsychischen Felde zu Grunde, ob derselbe in allen seinen Einzelheiten eine mechanische Folge sinnlicher Erregungen in den pathopsychischen Nervensystemen ist oder ob er nur zum Theil durch Sinnesempfindungen gestützt ist.

Aber wie gesagt, die Selbstbeoachtung lehrt, dass der blossen, sinnlich nicht gestützten Vorstellung eines Punktes im Raume jene Bestimmtheit fehlt, die der in allen Einzelheiten sinnlich gestützten Raumcomponente eigen ist. Nur wenn ein Punkt meiner Hautoberfläche berührt wird, ist das räumliche Moment dieser Sinneswahrnehmung ein völlig eindeutiges. Nichts an ihm lässt meinem Vorstellungsvermögen Spielraum.

1) Eine Bestätigung dieser Auffassung gewähren die klinischen Beobachtungen bei corticaler Tastlähmung. Solche Kranke können die einzelnen Tastreize sehr wohl in Bezug auf rechts-links, oben-unten, kurz in zwei Dimensionen anordnen. Sie können Ziffern oder Buchstaben, die man ihnen in die erkrankte Hand schreibt, tadellos erkennen. 
Sehe ich dagegen mit beiden Augen irgend ein Licht, so bleibt die Entfernung desselben von mir unbestimmt. Hierbei ist aber doch die Entfernungsvorstellung nicht völlig in mein Belieben gestellt; ich sehe, dass das Licht näher als dieses, entfernter als jenes Licht von mir sich befindet.

Beim einäugigen Sehen ist nur mehr bestimmt, ob das Object rechts, links, oben oder unten von anderen Gegenständen gelegen ist.

Bei der Wahrnehmung eines Tones, ist lediglich der Intensitätsunterschied in der Erregung beider Gehörorgane für das räumliche Moment bestimmend. Da es nur eine einfach unendliche Mannigfaltigkeit solcher Intensitätsunterschiede gibt, kann nur eine einfach quantitative Veränderung des räumlichen Momentes der Gehörwahrnehmung vorhanden sein. Und in der That vermögen wir nur zu unterscheiden, so lange wir uns nicht bewegen, d. h. solange wir allein auf das Gehörorgan angewiesen sind, ob sich ein akustisches Object rechts oder links befindet, bezw. mehr rechts oder mehr links als ein anderes.

Aber ebenso wie wir beim einäugigen Sehact bemerkten, dass wir die Objecte nicht etwa in einem zweidimensonalen Raum wahrnehmen, ebenso bemerken wir, dass auch beim Hören die durch die Wahrnehmung des akustischen Objectes erzeugte Ortsvorstellung eine dreidimensionale ist.

Nur der Grad der Bestimmtheit in den drei Raumerstreckungen wechselt bei der durch die versehiedenen Sinneswahrnehmungen erzeugten Ortvorstellung. Sofern diese nicht sinmlich gestützt ist, ist sie mehr weniger von unserem Willen abhängig, und kann, ohne dass der Sinnesreiz sich ändert, wechseln. Wir können uns ganz nach Belieben in Fig. $2 a b$ oder $c d$ als vordere Kante vorstellen. In irgend einem kleinsten Zeittheilchen aber können wir nur eine beider Vorstellungen haben. Wenn wir uns irgend einen Punkt im Raume denken, so wandert zwar dieser Punkt beständig, in jedem einzelnen Zeittheilchen aber ist er ein ganz bestimmter Punkt im Raum. Jede Raumvorstellung also besteht unbedingt aus drei psychischen Elementen, beruht also auf einer Geschwindigkeitsänderung der drei stereopsychischen Theilchemismen.

Rein theoretisch ist der Fall denkbar, dass ein Sinnesreiz einen, zwei oder alle drei Teilchemismen auf ganz bestimmte Geschwindigkeiten einstellt, dass er nur das Verhältniss der drei Geschwindig- 
Der Wille u. das räumliche Moment in Wahrnehmung u. Vorstellung. 335

keiten oder zweier zu einander bestimmt oder auch ihre absolute Grösse. Je nachdem erbält, wie wir geschildert haben, das räumliche Moment der Vorstellung in mehr oder weniger Elementen seine sinnlich gestützte Bestimmtheit.

$\mathrm{Ob}$ aber ein einziger solcher Teilchemismus oder alle durch die Erregung in einem Sinnescentrum auf bestimmte Geschwindigkeiten festgelegt werden, in jedem Falle besteht in jedem Augenblick für jeden dieser Chemismen eine gewisse Geschwindigkeit, und diesen Vorgang müssen wir als dreidimensionale Vorstellung erleben. Ist z. B. bei der zweiäugigen Sehwahrnehmung eines Lichtpunktes das Verhältniss $x: y$ festgelegt, so bedeutet $z$ eine Geschwindigkeit des dritten Chemismus, die nicht durch ausserhalb des stereopsychischen Feldes gelegene Bestimmungen geregelt ist. Der Werth von z ist abhängig von dem Zustande des stereopsychischen Feldes, indem es sich gerade unter allen berrschenden äusseren und inneren Einflüssen befindet, während der Werth des Verhältnisses $x: y$, solange der Sehreiz derselbe bleibt, von Veränderungen dieses Zustandes nicht beeinflusst wird. $\mathrm{Ob}$ wir beim einäugigen Sehen den Punkt $A$ rechts oder links von $B$ darüber oder darunter wahrnehmen, steht nicht in unserem Belieben. $\mathrm{Zu}$ einem Teil ist der stereopsychische Stoffwechsel - in diesem Falle - mechanisch bestimmt durch den äusseren Reiz, durch extrastereopsychische Kräfte. $\mathrm{Zu}$ einem anderen Theile aber ist es die mechanische Causalität der intrastereopsychischen Verhältnisse, welche die Stoffwechselgeschwindigkeiten bestimmt. Diesen Theil erleben wir als die von unserem Belieben oder Willen abhängige Vorstellung: $\mathrm{Ob}$ und wie weit Punkt $B$ bei einäugiger $\mathrm{Be}$ trachtung vor oder hinter $A$ gelegen ist.

Natürlich ist auch der Werth von $z$ in jedem Augenblick durch die geschlossene Kette der mechanischen Causalität bestimmt. Die Veränderungen im stereopsychischen Felde sind dieser Causalität ebenso unterworfen wie alle materiellen Processe überhaupt. Aber nur ein Theil dieser steropsychischen Processe steht zu einer bestimmten Zeit in gesetzmässiger Abhängigkeit von den durch Sinnesreize erzeugten Vorgängen in anderen Neuronsystemen. Ein anderer Theil ist als die hiervon unabhängige Lebensthätigkeit des stereopsychischen Feldes zu betrachten, und dieser Theil wird von uns als willkürlich, als in unserem Belieben stehend, erlebt.

Die unabhängig von dem Zustande der extrastereopsychischen 
Gehirntheile auf Grund intrastereopsychischer Causalität sich abspielenden Stoffwechseländerungen erleben wir als von unserem Willen abhängige Vorstellungen.

Der Wille ist das Erleben der mechanischen Causalität unseres Bewusstseinsorgans.

Alle nicht gewollten Vorstellungen entstehen und bestehen so lange und insofern, als der Stoffwechsel im stereopsychischen Felde bestimmt ist durch die Zustände in anderen Neuronsystemen.

Solange wir wachen, ist nun das stereopsychische Feld nie völlig unabhängig von den Vorgängen in den pathopsychischen Neuronsystemen. Wir können zwar uns durch einen Willensakt bis zu einem unglaublichen Grade gegen alle Sinneseindrücke abschliessen, indem wir uns in uns selbst versenken; stets aber bleibt von unserem Wollen unabhängig in uns vorbanden die Vorstellung des nach unserem Körper"orientirten Raumes mit seinen drei Dimensionen, "mit seinem Oben-Unten, Hinten-Vorn und Rechts-Links.

Diese Vorstellung ist durchaus nicht von unserer Willkür abhängig, sie ist der Bewusstseinswerth dafür, dass ständig von der Haut, den Gelenken und der Netzhaut aus Erregungen des stereopsychischen Feldes stattfinden ${ }^{1}$ ). Und das, was wir bisher als räumliches Moment der Wahrnehmung bezeichnet hatten, ist eigentlich nur dahin zu verstehen, dass ein bestimmter Theil dieser Gesammtvorstellung einen besonderen Bewusstseinswerth erhält, mit Aufmerksamkeit beachtet wird.

Es ist nicht zu bezweifeln, dass fortwährend, z. B. von dem kleinen Finger meiner Hand sensible Erregungen dem Hirne zufliessen. Aber für gewöhnlich achte ich nicht darauf. Die Aufmerksamkeit ist auf dieses Glied nicht gerichtet. In dem Augenblicke aber, wo ich meine Aufmerksamkeit auf dieses Glied lenke, wenn ich z. B. mit ihm einen Kreis beschreiben will, tritt sein Ort als Raumvorstellung klar in's Bewusstsein. Eine Veränderung des Sinnesreizes ist für diesen Act der Aufmerksamkeit ganz überflüssig. Ohne dass eine solche erfolgt, kann ich jeder Zeit - d. b. wenn ich will - auf irgend einen Körpertheil meine Aufmerksamkeit lenken.

1) Man beurtheile hier nach den tiefen Sinn, der in der Aufforderung liegt, man solle sich einmal selbst als nicht vorhanden vorstellen, um die "Realität" der objectiven Welt zu erkennen! 
Der Wille $u$, das räumliche Moment in Wahrnehmung u. Vorstellung. 337

Offenbar ist dann das stereopsychische Feld für eine bestimmte Erregung im centralen Tastfelde zugänglicher geworden, der adäquate Reiz im Tastcentrum kann nunmehr auf die Stereone überfliessen.

Handelt es sich - psychologisch gesprochen - um einen Act freiwilliger Aufmerksamkeit auf eine bestimmte Stelle meines Körpers, so kann diese Veränderung der Stereone nur aus ihrer Eigenthätigkeit erklärt werden. Werde ich in die Fingerspitze mit einer Nadel gestochen, so tritt dieselbe Aufmerksamkeit ein, aber aus extrastereopsychischen Ursachen. Die Erregung in einem Sinnescentrum steigt dann in dem Maasse, dass der Leitungswiderstand zum stereopsychischen Felde gesprengt wird.

Der stereopsychische Vorgang, die Ortsvorstellung eines Körpertheiles, ist in beiden Fällen derselbe. Nur wird er das erste Mal durch intrastereopsychische, das andere Mal durch extrastereopsychische Ursachen erzeugt. Das erste Mal bin ich thätig, das zweite Mal leidend.

Nimmt also aus irgend welchen im stereopsychischen Felde gelegenen Ursachen der Leitungswiderstand desselben gegen ein Sinnescentrum ab, findet also eine Erniedrigung der stereopsychischen Reizschwelle statt, so erlebe ich das als gewollte Vorstellung eines Körpertheiles.

Findet von einem Sinnescentrum aus aus irgend welchen anderen Ursachen, z. B. durch Anwachsen der Sinnesenergie, ein Uebergreifen der Erregung auf das stereopsychische Feld statt, so ist die Vorstellung, als welche ich den stereopsychischen Process erlebe, eine von meinem Willen unabhängige.

Ein je grösserer Teil der stereopsychischen Kraft von den pathopsychischen Erregungen festgelegt wird, ein desto geringeres Maass stereopsychischer Thätigkeit bleibt für die willkürlichen Vorstellungen frei.

Die Bewusstseinseigenheit, die wir als Aufmerksamkeit auf eine bestimmte Stelle unseres Körpers untersuchen, hängt also davon $a b$, dass ein in irgend einem Sinnescentrum vorhandener Erregungsvorgang zum Reiz für die Stereopsyche wird. Während für gewöhnlich, wenn ich durchaus nicht auf meinen Körper achte, alle sinnlichen Erregungen nur die allgemeine Raumvorstellung von Oben-Unten, Hinten-Vorn, Rechts-Links veranlassen, hebt sich aus dieser Resultantenvorstellung, aus allen von aussen auf die StereoE. Pflüger, Archiv für Physiologie. Bd. 95. 
psyche einwirkenden Reizen, im Augenblick, wo ich die Aufmerksamkeit auf einen Theil meines Körpers lenke, ein bestimmter Theil deutlich heraus. Es ist das räumliche Moment der Wahrnehmung im eigentlichen Sinne des Wortes.

Da die pathopsychischen Neuronsysteme an versehiedenen umgrenzten Theilen der Grosshirnrinde liegen, da Grund zu der Annahme vorliegt, dass die Haut der Hand an einer anderen Stelle der Hirnrinde ihr Projectionsfeld hat als die des Fusses, so würde bei einem Wandern der Aufmerksamkeit über die Körperoberfläche hin auch eine wandernde Veränderung des stereopsychischen Feldes stattfinden müssen. Die Herabsetzung der Reizschwelle müsste in jedem Augenblicke gerade die Stereone betreffen, welche mit den die gerade beachtete Hautstelle vertretenden Sinneszellen articuliren.

Da aber unsere Kenntniss von der Projection der Körperoberfläche in der Hirnrinde noch an Sicherheit zu wünschen übrig lässt, scheint es zweckmässig, den der Aufmerksamkeit zu Grunde liegenden Process noch von einer anderen Seite her zu untersucben.

Die Localisation der motorischen Centren der Hirnrinde ist bedeutend besser bekannt. Wenn ich meinen linken Arm, meinen Kopf oder meinen rechten Zeigefinger allein bewegen will, so kann

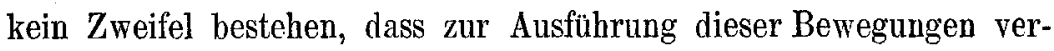
schieden localisirte motorische Rindenfoci innervirt werden müssen.

Will ich mit meinem linken kleinen Finger ein A schreiben und dann dieselbe Bewegung mit meiner Nasenspitze ausführen, so unterscheiden sich diese beiden Willenserscheinungen lediglich durch die verschiedene Oertlichkeit des Körpers, auf welche die Aufmerksamkeit gerichtet ist. Die Wegvorstellung ist in beiden Fällen die gleiche, nur die Lage des Weges im Raum, oder der zu bewegende Körpertheil ist verschieden.

Diese Verschiedenheit beider Bewusstseinsthatșachen äussert sich nun auch in einer localisatorischen Verschiedenheit der begleitenden Hirnvorgänge. Will ich den linken kleinen Finger bewegen, so fliesst die stereopsychische Erregung an einer anderen Stelle auf das motorische Neuronsystem über, als wenn ich die Nasenspitze bewegen will. Da ich diese Bewegungen ja will, d. $h$. da sie in meinem Belieben stehen, kann die Ursache der verschiedenen Abflussstellen stereopsychischer Energie nur in einer Veränderung des stereopsychischen Feldes selbst gesucht werden. 
Der Wille u. das räumliche Moment in Wahrnehmung u. Vorstellung. 339

An einer bestimmten Stelle des stereopsychischen Feldes, die verschieden ist, je nach dem zu bewegenden Körpertheil, steigt die Spannung der nervösen Energie, bis sie den Leitungswiderstand der articulirenden motorischen Neurone überwindet.

Würde aus irgend weichen Gründen die Reizsch welle im motorischen Neuronsystem der Hirnrinde an irgend einer Stelle so sinken, dass hier die stereopsychische Erregung überfliesst, so würden auch Bewegungen erfolgen, diese Bewegungen aber wären unwillkürliche, wie bei der Epilepsie oder manchen motorischen Erscheinungen bei der Hysterie und Geisteskrankheit').

In gewissen motorischen Neuronsystemen liegt die Reizschwelle offenbar physiologischer Weise schon niedriger als in anderen. Schon das kleine Kind greift eher und häufiger mit der rechten Hand zu als mit der linken. Sebr deutlich ist diese Herabsetzung der Reizschwelle der motorischen Rindenzellen in Fällen von Chorea ausgesprochen. In der mebr erkrankten Hand erfolgen bei Willkürbewegungen der gesunderen stets Mitbewegungen, um so deutlicher, je mehr man von dem kranken Gliede die Aufmerksamkeit ablenkt.

Wir sind nunmehr im Stande, uns ein Bild von den Gehirnvorgängen $z u$ machen, die wir als Willen zu einer bestimmten Willkürbewegung erleben. Intrastereopsychisch bedingte Vorgänge im stereopsychischen Feld, die wir als von unserem Willen abhängige Raumvorstellungen erleben, setzen an einem Theile des stereopsychischen Feldes, wo es an ein Sinnescentrum grenzt, die Reizschwelle herab, so dass die Erregung dieses Sinnescentrums ein wirksamer Reiz für die Stereone wird: Ich lenke meine Aufmerksamkeit auf einen bestimmten Körpertheil. Zu gleicher Zeit aber steigt die Spannung der nervösen Energie an derjenigen Stelle des stereopsychischen Feldes, welches an die, die Bewegungen des beachteten Körpertheiles beherrschenden, motorischen Rindenzellen angrenzt.

Sind nunmehr in dem Zustande des stereopsychischen Feldes keine Gründe für eine Veränderung der intrastereopsychischen Energievertheilung vorhanden, so wollen wir den betreffenden Körpertheil nicht bewegen, und objectiv nachweisbar äussert sich diese Aufmerksamkeit in einer muskulären Feststellung des Gliedes. Der

1) Solche Bewegungen müssten dem Kranken wie durch eine fremde Gewalt entstanden erscheinen. 
Muskeltonus steigt. Sind aber die Energieverhältnisse im stereopsychischen Felde so beschaffen, dass eine Veränderung eintreten muss in den zur Entladung vorbereiteten Stereonen, so tritt eine Willkürbewegung des beachteten Körpertheiles ein. Eine solche Beschaffenheit der stereopsychischen Energieverhältnisse hat als Bewusstseinswerth die Vorstellung des Weges, den der Körpertheil beschreiben soll.

Ist diese Wegvorstellung eine einfache Richtung, will ich den rechten Arm seitlich, nach vorn, oder in irgend einer der möglichen Zwischenrichtungen erheben, so ist in jedem Zeittheilchen das Geschwindigkeitsverhältniss der drei stereopsychischen Elementarchemismen in den die motorischen Zellen innervirenden Stereonen dasselbe $x: y: z$, und dem entsprechend sind auch während der Bewegung die im Schultergelenk wirksamen drei Bewegungskräfte dauernd im Verhältniss $x: y: z$ thätig.

Ist die Bewegung zu Ende, habe ich den Arm so boch gehoben wie ich will, so ist die Gesammtgeschwindigkeit der drei stereopsychischen Elementarchemismen der neuen Lagevorstellung des Armes entsprechend eine andere, und zwar ist der Unterschied der Gesammtgeschwindigkeit am Anfang und Ende der Bewegung eine Function der absoluten Grösse der Wegvorstellung.

Würde ich z. B. allein mit dem Zeigefinger eine Kreisbewegung ausführen wollen, so würden die mit dem motorischen Focus des Zeigefingers artikulirenden Stereone, die "Actionsstereone" folgendes Verhalten zeigen: Sei das Verhältniss der drei stereopsychischen Elementarchemismen in ihnen am Anfange der Bewegung $x: y: z$, so würde das Verhalten $x: y$ alle möglichen Werthe durchlaufen, während sowohl die Summe $x+y$, wie das Verhältniss $(x+y): z$ constant bleibt.

Demgemäss würden die das Grundgelenk des Zeigefingers bewegenden zwei Kräfte der Spreizung und Beugung-Streckung in jedem Augenblick dieselbe Summe haben, ihr gegenseitiges Verhältniss aber würde nach einander alle möglichen Werthe durchlaufen.

Zur Ausführung einer dreidimensionalen Bewegung aber würden die drei stereopsychischen Elementarkräfte ihr gegenseitiges Verhältniss ändern müssen, und würden dementsprechend mindestens drei von einander unabhängige Bewegungsmechanismen in wechselnder Stärke in Thätigkeit versetzen. 
Würden nun die Bewegungen unserer Glieder in jeder beliebigen Richtung den gleichen Wiederstand finden, $\therefore$ würde dieselbe Kraftgrösse, welche meinen Zeigefinger im Grundgelenk um $20^{\circ}$ beugt, ihn auch um $20^{\circ}$ spreizen, so würden wir alle ohne Weiteres im Stande sein, jede Bewegungsvorstellung auch richtig auszuführen. In der That aber sind die Widerstände für unsere Bewegungen in den verschiedenenRichtungen sehr ungleich. Man denke, dass die Schwerkraft bald hemmend, bald fördernd wirken kann, dass nicht eine Gelenkfläche existirt, die im mathematischen Sinne ein Rotationskörper wäre, und man wird begreifen, dass ein ständig wechselndes Verhältnis der Beugung-Streckungs- und Spreizungskraft bei gleichbleibender Stärke der Gesammtinnervation den Zeigefinger nicht auf einem Kegelmantel bewegen kann. Keine unserer Bewegungen dürfte daher mit der uns geläufigen Genauigkeit ausgeführt werden, wenn mit der geschilderten stereopsychischen Veränderung der Hirnprocess bei der Willkürbewegung wirklich erschöpft wäre.

Wir würden unser Leben lang ataktisch sein, wie Neugeborene und kleine Kinder es sind.

Aber während der Bewegungen und dureh sie finden Sinnesreize statt, die ein räumliches Moment haben. Schreibe ich bei geschlossenen Augen einen Kreis mit dem Zeigefinger in die Luft, so sind es wesentlich die Elemente der tiefen Sensibilität, die Gelenksnerven, welche gereizt werden. Das räumliche Moment dieser Empfindungen ist jedenfalls nicht in allen seinen Einzelheiten sinnlich gestützt, ein grosser Teil desselben ist meinem Belieben überlassen, und in meinen Belieben liegt in diesem Falle die Kreisvorstellung. Ich empfinde keinen Widerspruch zwischen dem räumlichen Moment des Bewegungswillens und dem der Bewegungswahrnehmung.

Zeichne ich aber den Kreis bei geschlossenen Augen mit der Zeigefingerspitze in die Hohlhand, so bemerke ich unter Umständen, dass die Bewegung nicht ganz richtig ist. Das räumliche Moment der Bewegungswahrnehmung ist viel vollkommener sinnlich gestützt, d. h. von meinem Willen weniger abhängig als im ersten Falle, und weicht in etwas von dem des Bewegungswillens ab.

Diese Abweichung wird um so deutlicher, je vollkommener die sinnliche Stütze des räumlichen Momentes der Bewegungswahrnehmung wird, also beim Sehen des durchlaufenen Weges, und erreicht die grösste Deutlichkeit, wenn alle Theile dieser Wegwahr- 
nehmung gleichzeitig sinnlich gestützt werden, wenn ich z. B. den Kreis mit Kreide an die Tafel zeichne.

In jedem Falle findet also durch die willkürliche Bewegung von den Sinnescentren her eine stereopsychische Erregung statt, die uns als räumliches Moment der Bewegungswahrnehmung bewusst wird. Ist dieses räumliche Moment der Bewegungswahrnehmung nicht merkbar verschieden von dem des Bewegungswillens, so war die Bewegung "zweckmässig", wir fühlen uns befriedigt.

Weichen beide Wegvorstellungen merklich von einander $a b$, so erleben wir ein Gefühl der Unlust.

Nach unseren Ausführungen ist die Vorstellung des Kreises in unserem Beispiel eine willkürliche, d. h. sie ist der Bewusstseinsausdruck für einen materiellen Vorgang, der sein Dasein lediglich intrastereopsychischen Kräften verdankt. Jede andere Vorstellung, sofern sie auf extrastereopsychische Erregungen zurückgeht, also z. B. durch Sinnesreize erzeugt wird, ist also ein Hemmniss des nach intrastereopsychischen Gesetzen ablaufenden Geschehens.

Ist die Energie des intrastereopsychischen Vorganges eine grosse d. h. halte ich mit grosser Willenskraft die Wegvorstellung fest, so vermag das räumliche Moment der Bewegungswahrnehmung diese nicht zu verdrängen, aber die Unvereinbarkeit der beiden räumlichen Vorstellungen wirkt unangenehm. Dem augenblicklichen Zustande der Stereopsyche gemäss ist nur der der gewollten Vorstellung entsprechende Vorgang; und die ungewollte der Bewegungswahrnebmung bedeutet nur die Nothwendigkeit eines grösseren intrastere0psychischen Kraftaufwandes.

Wozu wird diese grössere stereopsychische Kraftleistung verwandt, wie verräth sie sich im Bewusstsein? Auf das unangenehme Gefühl, von dem die Wahrnehmung einer fehlerhaften Bewegung begleitet ist, habe ich schon hingewiesen, so dass wir berechtigt sind, dieses Unlustgefühl als das Erlebniss extrastereopsychischer Einflüsse zu bezeichnen, welche den freien Ablauf der intrastereopsychischen Vorgänge entgegen wirken, oder als Reizwirkungen, die dem jeweiligen Zustande der Stereopsyche schädlich sind, insofern sie einen nicht ökonomischen Mehrverbrauch von stereopsychischen Kräften erzeugen, oder die Lebensenergie unseres Bewusstseinsorganes schädigen.

Besteht aber der feste Wille, die Wegvorstellung genau aus- 
Der Wille u. das räumliche Moment in Wahrnehmung u. Vorstellung. 343

zuführen, so zeigt sich bei jeder Wiederholung der Bewegung eine Verminderung der Fehler, bis schliesslich die Bewegungswahrnehmung in ihrer Raumcomponente identisch geworden ist mit der des Bewegungswillens. Wir schliessen daraus, dass jede Wahrnehmung eines Fehlers in der Ausführung der Bewegung, den motorischen Apparat, zunächst das motorische Neuronsystem der Hirnrinde, in gewisser Weise verändern muss, und diese Veränderung besteht eben darin, dass allmählich nur die Nervenbahnen beschritten werden, nur die Muskeln sich zusammenziehen, welche, indem sie durch die Bewegung die Sinnesorgane reizen, im stereopsychischen Felde den auf Grund der in ihm wirkenden Kräfte vorhandenen Zustand nicht stören, sondern fördern. Diese allmähliche Veränderung des corticalen und subcorticalen motorischen Apparates bis hinab zu den Muskeln und Knochen ist die weitere Wirkung des Mehraufwandes stereopsychischer Energie bei Wahrnehmung fehlerhafter, d. i. ungewollter, Bewegungen: „Es ist der Geist, der sich den Körper baut."

Der individuelle Wille hat seine Grenzen in dem beschränkten Kraftmaasse des Individuums, der phyletische ist grenzenlos wie die Zeit in seiner Macht.

Es ist ein verhältnissmässig beschränktes Gebiet geistiger Thätigkeit, dass ich in den vorliegenden Blättern der Analyse unter zogen habe. Soweit sich diese Analyse auf Bewusstseinserscheinungen bezieht, glaube ich mich von nicht erweisbaren Theorien frei gehalten zu haben, es handelt sich um einfache Beschreibungen von Thatsachen. Soweit ich versuchte, diesen Bewusstseinserscheinungen eine materielle, in die Kette der mechanischen Causalität eingeschaltete Grundlage zu geben, ist alles bypothetisch. Aber diese Hypothese ist so lange brauchbar, als sie mit den Thatsachen im Einklange steht, und sie erscheint mir nothwendig, weil wir an der Hand einer solchen Hypothese die Gesetzmässigkeit unserer Bewusstseinserscheinungen verstehen und prüfen können. Nur die mechanische Causalität macht uns verständlich, warum A sein muss, wenn B. ist.

Nochmals möchte ich die Hauptgedanken, die im Vorstehenden entwickelt wurden, zusammenfassen:

Das stereopsychische Feld besitzt einen aus drei Theilchemismen bestehenden Stoffwechsel. Alle Veränderungen, welche dieser Stoffwechsel erleiden kann, bestehen in einer Geschwindigkeitsänderung eines, zweier oder aller drei Chemismen, die entweder alle Stereone oder nur einen Theil derselben betreffen. 
Nie, so lange wir wachen, ist die Thätigkeit der Stereopsyche vollkommen unabhängig von extrastereopsychischen Kräften, von den in den Sinnescentren stattfindenden Erregungen. Diese Erregungen geben uns das Bewusstsein des nach unserem Körper orientirten Raumes, und kein Sinnesreiz vermag etwas anderes als irgend einen Theil dieses Raumes in das Blickfeld der Aufmerksamkeit zu heben.

Diese Aufmerksamkeit auf eine bestimmte Stelle im Raum ist das Anzeichen für eine bestimmte Localisation der stereopsychischen Veränderung. Erfolgt diese besondere Localisation auf Grund intrastereopsychischer Kräfte, so erscheint sie freiwillig, erfolgt sie durch Sinnesreize, so erscheint sie von unserem Willen unabhängig.

Jedes räumliche Moment einer Sinneswahrnehmung bedeutet also, durch welches Sinnesorgan es immer erzeugt sei, an bestimmter Stelle des stereopsychischen Feldes eine bestimmte Veränderung in der Geschwindigkeit des Stoffwechsels gegen die Ausgangsgesch windigkeit, welche wir als Vorstellung des nach unserem Körper orientirten Raumes erleben, der das Maass für alle übrigen Raumvorstellungen abgibt. Verändert sich der gesammte stereopsychische Stoffwechsel in allen seinen Theilen, nimmt z. B. die Gesammtgeschwindigkeit des Stoffwechsels in allen Theilen zu unter Wahrung des Verhälthältnisses $x: y: z$, so nehmen wir eine Bewegung unseres ganzen Körpers in einer Richtung wahr, eine Empfindung, die ebensowohl von dem Gesichtssinn wie vom Ohrlabyrinth, wie auch von fast allen übrigen Sinnesorganen erzeugt werden kann.

Jede Wahrnehmung der Bewegung eines Körperabsehnittes bedeutet eine besondere auf einen beschränkten Theil des stereopsychischen Feldes begrenzte Veränderung. Aber diese Veränderung beruht auf der Beschleunigung derselben Chemismen, ob sie hier oder dort stattfindet, auf einer Wirkung derselben Kräfte in unserem Bewusstseinsorgan.

Ein Theil der stereopsychischen Vorgänge ist aber stets unabhängig von den extrastereopsychischen Vorgängen im Centralnervensystem. Diese Vorgänge haben in unserem Bewusstsein keine Beziehungen zu dem nach unserem Körper orientirten Raum und sind nicht mehr räumliche Vorstellungen sondern Formbegriffe.

Der Begriff des Kreises, der Geraden bleibt von jeder Localisation unabhängig, ebenso von jeder absoluten Grössenvorstellung. Der Kreis kann überall im Raume sein, aber sobald er irgend an einer 
Der Wille u. das räumliche Moment in Wahrnehmung u. Vorstellung. 345 bestimmten Stelle ist, ist er nicht mehr Kreisbegriff, sondern Vorstellung; der vorher diffuse stereopsychische Process hat sich an bestimmter Stelle des stereopsychischen Feldes verdichtet.

$\mathrm{Ob}$ aber die Veränderungen des stereopsychischen Feldes aus inneren oder äusseren Ursachen erfolgen, immer können sie im Bewusstsein nur als eine der möglichen Combinationen aus 3 Elementen, als Raum, Fläche oder Linie erscheinen, als Richtungscomplexe 1, 2 oder 3 dimensionaler Natur. 